\title{
Analysis of flexural wave propagation in poroelastic composite hollow cylinder
}

\author{
J. Manoj Kumar ${ }^{1}$, S. Ahmed Shah ${ }^{2 *}$, C. Nageswara Nath ${ }^{3}$ \\ ${ }^{I}$ Department of Mathematics, BVRIT College of Engineering for Women, Hyderabad - 500 090, INDIA \\ ${ }^{2}$ Department of Mathematics, Deccan college of Engineering and Technology, Hyderabad-500 001, INDIA \\ ${ }^{3}$ Department of Mathematics, CMR Technical Campus, Hyderabad - 501 401, INDIA \\ "Corresponding author, E-mail address: ahmed_shah67@yahoo.com )
}

\begin{abstract}
Wave propagation in an infinitely long poroelastic composite hollow cylinder in is examined by employing Biot's theory of wave propagation in poroelastic media. A poroelastic composite hollow cylinder consists of two concentric poroelastic cylindrical layers both of which are made of different poroelastic materials with each poroelastic material as homogeneous and isotropic. The inner and outer boundaries of composite hollow poroelastic cylinder are free from stress. The frequency equation of flexural vibrations of poroelastic composite hollow cylinder is obtained. In addition some particular cases such as poroelastic composite hollow cylinder with rigid casing, poroelastic composite bore and poroelastic bore are discussed. Non-dimensional phase velocity is computed as a function of non-dimensional wavenumber. The results are presented graphically for two types of poroelastic composite cylinders and then discussed.
\end{abstract}

Key words: Biot's theory, poroelastic composite hollow cylinder, wavenumber, rigidity, phase velocity

DOI: http://dx.doi.org/10.4314/ijest.v8i1.2

\section{Introduction}

Gazis (1959) studied the propagation of free harmonic waves in elastic hollow circular cylinder. McNiven et al. (1963) discussed propagation of axially symmetric waves in composite elastic rods. Soldatos and Hadjigeorgiou (1990) have obtained a threedimensional solution for free vibration problem of homogeneous isotropic cylindrical shells and panels. Cui et al. (1997) and Abousleiman and Cui (1998) presented poroelastic solutions in an inclined borehole and transversely isotropic well-bore cylinders. Ahmed shah and Tajuddin (2009) discussed axially symmetric vibrations of finite composite poroelastic cylinders. Malla Reddy and Tajuddin (2010) studied axially symmetric vibrations of composite poroelastic cylinders. Sharma and Sharma (2010) analyzed free vibration in a homogeneous transradially isotropic thermoelastic sphere. Flexural wave propagation in coated poroelastic cylinders is presented by Ahmed shah (2011). Tajuddin (2011) et al. discussed axial shear vibrations in a poroelastic composite cylinder. Shanker et. al. (2012) studied radial vibrations in an infinitely long poroelastic composite cylinder.

In the present analysis, flexural vibrations in poroelastic composite hollow cylinder are investigated employing Biot's (1956) theory of wave propagation in porous materials. Biot's model consists of an elastic matrix permeated by a network of interconnected spaces saturated with liquid. The frequency equations of flexural vibrations are obtained for poroelastic composite hollow cylinder and as well for some particular cases i.e., poroelastic composite hollow cylinder with rigid casing, poroelastic composite bore and poroelastic bore each for pervious and impervious surfaces. Non-dimensional phase velocity as a function of non-dimensional wavenumber is computed in each case. The results are presented graphically for two types of poroelastic composite cylinders and then discussed. The poroelastic constants of only two poroelastic materials (1. Sandstone saturated with water and 2. Sandstone saturated with kerosene) are available. So, the numerical work has been done only on these two materials. 


\section{Governing Equations, formulation and solution of the problem}

The equations of motion of a homogeneous, isotropic poroelastic solid (Biot 1956) in the presence of dissipation $b$ are:

$$
\begin{gathered}
N \nabla^{2} \mathbf{u}+(A+N) \nabla e+Q \nabla \varepsilon=\frac{\partial^{2}}{\partial t^{2}}\left(\rho_{11} \mathbf{u}+\rho_{12} \mathbf{U}\right)+b \frac{\partial}{\partial t}(\mathbf{u}-\mathbf{U}) \\
Q \nabla e+R \nabla \varepsilon=\frac{\partial^{2}}{\partial t^{2}}\left(\rho_{12} \mathbf{u}+\rho_{22} \mathbf{U}\right)-b \frac{\partial}{\partial t}(\mathbf{u}-\mathbf{U})
\end{gathered}
$$

where $\nabla^{2}$ is the Laplacian operator $\mathbf{u}(\mathrm{u}, \mathrm{v}, \mathrm{w})$ and $\mathbf{U}(\mathrm{U}, \mathrm{V}, \mathrm{W})$ are solid and liquid displacements; e and $\varepsilon$ are the dilatations of solid and liquid. $A, N, Q, R$ are all poroelastic constants and $\rho_{11}, \rho_{12}, \rho_{22}$ are the mass coefficients following Biot (1956) such that the sums $\left(\rho_{11}+\rho_{12}\right)$ and $\left(\rho_{12}+\rho_{22}\right)$ are masses of solid and liquid, respectively, the parameter $\rho_{12}$ represents mass coupling between solid and liquid. The poroelastic constants $A$ and $N$ correspond to familiar Lame' constants in a purely elastic solid. The coefficient $N$ represents the shear modulus of the solid. The coefficient $R$ is a measure of the pressure required on the liquid to force a certain amount of the liquid into the aggregate while the total volume remains constant. The coefficient $Q$ represents the coupling between the volume changes of solid to that of liquid.

The stresses $\sigma_{k l}$ and the liquid pressure $s$ of the poroelastic solid are

$$
\begin{gathered}
\sigma_{k l}=2 N e_{k l}+(A e+Q \varepsilon) \delta_{k l}, \quad(k, l=r, \theta, z) \\
s=Q e+R \varepsilon,
\end{gathered}
$$

where $\delta_{k d}$ is the well-known Kronecker delta function and $e_{k}$ are strain components of poroelastic solid.

Let $(r, \theta, \mathrm{z})$ be cylindrical polar co-ordinates. Consider a poroelastic composite hollow cylinder whose inner (core) and outer (casing) shells are made of different poroelastic materials with each poroelastic material as homogeneous and isotropic and whose axis is in the direction of z-axis. The inner radius of core is $r_{1}$, outer radius of casing is $r_{2}$ and ' $a$ ' is the interface radius. The prefixes $j=1,2$ are used to denote two cylinders related to poroelastic composite cylinder. The quantities with prefix (1) refer to the core, while the prefix (2) refers to the casing.

The displacements of solid ${ }_{j} \mathbf{u}\left({ }_{j} u,{ }_{j} v,{ }_{j} w\right)$ which can readily be evaluated from field equation (1) are

$$
\begin{aligned}
{ }_{j} u= & {\left[{ }_{j} C_{1 j} M_{51}(r)+{ }_{j} C_{2 j} M_{52}(r)+{ }_{j} C_{3 j} M_{53}(r)+{ }_{j} C_{4 j} M_{54}(r)+{ }_{j} C_{5 j} M_{55}(r)+{ }_{j} C_{6 j} M_{56}(r)+\right.} \\
& \left.{ }_{j} C_{7 j} M_{57}(r)+{ }_{j} C_{8} M_{58}(r)\right] \cos \theta e^{i(k z+\omega t)}, \\
{ }_{j} v= & {\left[{ }_{j} C_{1 j} M_{61}(r)+{ }_{j} C_{2}{ }_{2} M_{62}(r)+{ }_{j} C_{3 j} M_{63}(r)+{ }_{j} C_{4 j} M_{64}(r)+{ }_{j} C_{5 j} M_{65}(r)+{ }_{j} C_{6 j} M_{66}(r)+\right.} \\
& \left.{ }_{j} C_{7 j} M_{67}(r)+{ }_{j} C_{8 j} M_{68}(r)\right] \sin \theta e^{i(k z+\omega t)}, \\
j_{j} w= & {\left[{ }_{j} C_{1 j} M_{71}(r)+{ }_{j} C_{2 j} M_{72}(r)+{ }_{j} C_{3 j} M_{73}(r)+{ }_{j} C_{4 j} M_{74}(r)+{ }_{j} C_{5 j} M_{75}(r)+{ }_{j} C_{6 j} M_{76}(r)+\right.} \\
& \left.{ }_{j} C_{7 j} M_{77}(r)+{ }_{j} C_{8} M_{j} M_{78}(r)\right] \cos \theta e^{i(k z+\omega t)},
\end{aligned}
$$

where

$$
\begin{aligned}
{ }_{j} M_{51}(r) & =\frac{1}{r} J_{1}\left({ }_{j} \xi_{1} r\right)-{ }_{j} \xi_{1} J_{2}\left({ }_{j} \xi_{1} r\right) \\
{ }_{j} M_{55}(r) & =\frac{1}{r} J_{1}\left({ }_{j} \xi_{3} r\right) \\
{ }_{j} M_{57}(r) & =2 i k J_{2}\left({ }_{j} \xi_{3} r\right)
\end{aligned}
$$




$$
\begin{aligned}
& { }_{j} M_{61}(r)=-\frac{1}{r} J_{1}\left({ }_{j} \xi_{1} r\right) \\
& { }_{j} M_{65}(r)=-\frac{1}{r} J_{1}\left({ }_{j} \xi_{3} r\right)+{ }_{j} \xi_{3} J_{2}\left({ }_{j} \xi_{3} r\right) \\
& { }_{j} M_{67}(r)=2 i k J_{2}\left({ }_{j} \xi_{3} r\right) \\
& { }_{j} M_{71}(r)=i k J_{1}\left({ }_{j} \xi_{1} r\right) \\
& { }_{j} M_{75}(r)=0 \\
& { }_{j} M_{77}(r)=-{ }_{j} \xi_{3} J_{1}\left({ }_{j} \xi_{3} r\right)
\end{aligned}
$$

and ${ }_{j} C_{1},{ }_{j} C_{2},{ }_{j} C_{3},{ }_{j} C_{4},{ }_{j} C_{5}$ and ${ }_{j} C_{6}$ are constants, $\omega$ is frequency of wave, $k$ is wavenumber, $J_{\mathrm{n}}$ and $Y_{\mathrm{n}}$ are Bessel functions of first and second kind, respectively, each of order $n$,

$$
\begin{aligned}
& { }_{j} \xi_{l}^{2}=\frac{\omega^{2}}{{ }_{j} V_{l}^{2}}-k^{2}, \text { for } l=1,2,3, \\
& { }_{j} \delta_{k}^{2}=\frac{\left({ }_{j} P_{j} R-{ }_{j} Q^{2}\right)-{ }_{j} V_{1}^{2}\left({ }_{j} R{ }_{j} M_{11}-{ }_{j} Q_{j} M_{12}\right)}{{ }_{j} V_{k}\left({ }_{j} R{ }_{j} M_{12}-{ }_{j} Q_{j} M_{22}\right)} \text { for } k=1,2 \\
& { }_{j} M_{11}={ }_{j} \rho_{11}-\frac{i b}{\omega},{ }_{j} M_{12}={ }_{j} \rho_{12}+\frac{i b}{\omega},{ }_{j} M_{22}={ }_{j} \rho_{22}-\frac{i b}{\omega},
\end{aligned}
$$

${ }_{j} V_{1},{ }_{j} V_{2}$ are dilatational wave velocities of first and second kind, respectively, and ${ }_{j} V_{3}$ is shear wave velocity.

By substituting the displacements in equations (2), the relevant stresses pertaining to outer cylinder and inner cylinder are

$$
\begin{aligned}
& j_{j}\left(\sigma_{r r}+s\right)=\left[{ }_{j} C_{1 j} M_{11}(r)+{ }_{j} C_{2 j} M_{12}(r)+{ }_{j} C_{3 j} M_{13}(r)+{ }_{j} C_{4 j} M_{14}(r)+{ }_{j} C_{5 j} M_{15}(r)+{ }_{j} C_{6 j} M_{16}(r)+\right. \\
& \left.{ }_{j} C_{7 j} M_{17}(r)+{ }_{j} C_{8} M_{18}(r)\right] \cos \theta e^{i(k z+\omega t)}, \\
& { }_{j} C_{1 j} M_{21}(r)+{ }_{j} C_{2 j} M_{22}(r)+{ }_{j} C_{3 j} M_{23}(r)+{ }_{j} C_{4 j} M_{24}(r)+{ }_{j} C_{5 j} M_{25}(r)+{ }_{j} C_{6 j} M_{26}(r)+ \\
& \left.{ }_{j} C_{7 j} M_{27}(r)+{ }_{j} C_{8}\right)=\left[{ }_{j} C_{1 j} M_{28}(r)\right] \sin \theta e^{i(k z+\omega t)}, \\
& \left.{ }_{j} C_{7 j} M_{37}(r)+{ }_{j} C_{2} M_{j} M_{32}(r)+{ }_{j} C_{3 j} C_{8 j} M_{33} M_{38}(r)\right] \cos \theta e^{i(k z+\omega t)}, \\
& { }_{j} s=\left[{ }_{j} C_{4 j} M_{34} C_{1 j} M_{41}(r)+{ }_{j} C_{2 j} M_{42}(r)+{ }_{j} C_{3 j} C_{5 j} M_{43}(r)+{ }_{j} C_{4 j} M_{35}(r)+{ }_{j} C_{64}(r)\right] \cos \theta e^{i(k z+\omega t)}, \\
& \left(\frac{\partial s}{\partial r}\right)=\left[{ }_{j} C_{1 j} N_{41}(r)+{ }_{j} C_{2} N_{j} N_{42}(r)+{ }_{j} C_{3 j} N_{43}(r)+{ }_{j} C_{4 j} N_{44}(r)\right] \cos \theta e^{i(k z+\omega t),},
\end{aligned}
$$

where

$$
\begin{aligned}
& { }_{j} M_{11}(r)=\left\{\left[\left({ }_{j} Q+{ }_{j} R\right)_{j} \delta_{1}^{2}-\left({ }_{j} A+{ }_{j} Q\right)\right] k^{2}+\left[\left({ }_{j} Q+{ }_{j} R\right)_{j} \delta_{1}^{2}-\left({ }_{j} P+{ }_{j} Q\right)\right] \xi_{1}^{2}\right\} J_{1}\left({ }_{j} \xi_{1} r\right)+\frac{2{ }_{j} N_{j} \xi_{1}}{r} J_{2}\left({ }_{j} \xi_{1} r\right) \\
& { }_{j} M_{15}(r)=\frac{-2{ }_{j} N_{j} \xi_{3}}{r} J_{2}\left({ }_{j} \xi_{3} r\right)
\end{aligned}
$$




$$
\begin{aligned}
& { }_{j} M_{17}(r)=2 i_{j} N k_{j} \xi_{3} J_{1}\left({ }_{j} \xi_{3} r\right) \\
& { }_{j} M_{31}(r)=\frac{2{ }_{j} N i k}{r} J_{1}\left({ }_{j} \xi_{3} r\right)-2{ }_{j} N i k{ }_{j} \xi_{1} J_{2}\left({ }_{j} \xi_{1} r\right) \\
& { }_{j} M_{21}(r)=\frac{2{ }_{j} N_{j} \xi_{1}}{r} J_{2}\left({ }_{j} \xi_{1} r\right) \\
& { }_{j} M_{35}(r)=\frac{{ }_{j} N i k}{r} J_{1}\left({ }_{j} \xi_{3} r\right) \\
& { }_{j} M_{23}(r)=\frac{2{ }_{j} N_{j} \xi_{2}}{r} J_{2}\left({ }_{j} \xi_{2} r\right) \\
& { }_{j} M_{37}(r)=\frac{-{ }_{j} N_{j} \xi_{3}}{r} J_{1}\left({ }_{j} \xi_{3} r\right)+{ }_{j} N\left({ }_{j} \xi_{3}^{2}-k^{2}\right) J_{2}\left({ }_{j} \xi_{3} r\right) \\
& { }_{j} M_{25}(r)={ }_{j} N_{j} \xi_{1}^{2} J_{1}\left({ }_{j} \xi_{3} r\right)-\frac{2{ }_{j} N_{j} \xi_{3}}{r} J_{2}\left({ }_{j} \xi_{3} r\right){ }_{j} M_{41}(r)=\left({ }_{j} R_{j} \delta_{1}^{2}-{ }_{j} Q\right)\left(k^{2}+{ }_{j} \xi_{1}^{2}\right) J_{1}\left({ }_{j} \xi_{1} r\right) \\
& { }_{j} M_{27}(r)={ }_{j} N i k_{j} \xi_{3} J_{1}\left({ }_{j} \xi_{3} r\right) \\
& { }_{j} M_{45}(r)=0 \\
& { }_{j} M_{47}(r)=0
\end{aligned}
$$

${ }_{j} M_{i 2}(r)={ }_{j} M_{i 1}(r)$ for $\mathrm{i}=1,2,3,4,5,6,7$ with replacing $J_{n}$ and its derivatives, respectively,

by $Y_{n}$ and its derivatives,

${ }_{j} M_{i 4}(r)={ }_{j} M_{i 3}(r)$ for $\mathrm{i}=1,2,3,4,5,6,7$ with replacing $J_{n}$ and its derivatives, respectively,

by $Y_{n}$ and its derivatives,

${ }_{j} M_{i 6}(r)={ }_{j} M_{i 5}(r)$ for $\mathrm{i}=1,2,3,4,5,6,7$ with replacing $J_{n}$ and its derivatives, respectively,

by $Y_{n}$ and its derivatives,

${ }_{j} M_{i 8}(r)={ }_{j} M_{i 7}(r)$ for $\mathrm{i}=1,2,3,4,5,6,7$ with replacing $J_{n}$ and its derivatives, respectively,

by $Y_{n}$ and its derivatives,

$$
\begin{aligned}
{ }_{j} N_{41}(r) & =\frac{\left({ }_{j} R_{j} \delta_{1}^{2}-{ }_{j} Q\right)\left(k^{2}+{ }_{j} \xi_{1}^{2}\right)}{r} J_{1}\left({ }_{j} \xi_{1} r\right)-{ }_{j} \xi_{1}\left({ }_{j} R_{j} \delta_{1}^{2}-{ }_{j} Q\right)\left(k^{2}+{ }_{j} \xi_{1}^{2}\right) J_{2}\left({ }_{j} \xi_{1} r\right) \\
{ }_{j} N_{45}(r) & =0 \\
{ }_{j} N_{47}(r) & =0
\end{aligned}
$$

\section{Boundary conditions and frequency equation}

We assume that the outer surface of casing and inner surface of core are free from stress and there is a perfect bonding at the interface, thus the boundary conditions for stress-free vibrations of a poroelastic composite hollow cylinder in case of a pervious surface are

$$
\begin{aligned}
& \text { at } r=r_{1} ; \quad{ }_{1}\left(\sigma_{r r}+s\right)=0,{ }_{1}\left(\sigma_{r z}\right)=0,{ }_{1}\left(\sigma_{r \theta}\right)=0 \\
& \text { at } r=r_{2} ;{ }_{2}\left(\sigma_{r r}+s\right)=0, \quad{ }_{2}\left(\sigma_{r z}\right)=0, \quad{ }_{2}\left(\sigma_{r \theta}\right)=0 \\
& \text { at } r=a ;{ }_{1}\left(\sigma_{r r}+s\right)={ }_{2}\left(\sigma_{r r}+s\right),{ }_{1}\left(\sigma_{r z}\right)={ }_{2}\left(\sigma_{r z}\right),{ }_{1}\left(\sigma_{r \theta}\right)=0,{ }_{1} u={ }_{2} u \text {, } \\
& { }_{1} v={ }_{2} v,{ }_{1} w={ }_{2} w \\
& \text { at } r=r_{1}, r_{2} \text { and } a ; \quad{ }_{1} s={ }_{2} s=0,
\end{aligned}
$$

while the boundary conditions in case of an impervious surface are

$$
\begin{aligned}
& \text { at } r=r_{1} ; \quad{ }_{1}\left(\sigma_{r r}+s\right)=0,{ }_{1}\left(\sigma_{r z}\right)=0,{ }_{1}\left(\sigma_{r \theta}\right)=0 \\
& \text { at } r=r_{2} ; \quad{ }_{2}\left(\sigma_{r r}+s\right)=0,{ }_{2}\left(\sigma_{r z}\right)=0,{ }_{2}\left(\sigma_{r \theta}\right)=0
\end{aligned}
$$




$$
\begin{gathered}
\text { at } r=a ; \quad{ }_{1}\left(\sigma_{r r}+s\right)={ }_{2}\left(\sigma_{r r}+s\right),{ }_{1}\left(\sigma_{r z}\right)={ }_{2}\left(\sigma_{r z}\right),{ }_{1}\left(\sigma_{r \theta}\right)=0,{ }_{1} u={ }_{2} u, \\
{ }_{1} v={ }_{2} v, \quad{ }_{1} w={ }_{2} w \\
\text { at } r=r_{1}, r_{2} \text { and } a ; \quad \frac{\partial_{1} s}{\partial r}=\frac{\partial_{2} s}{\partial r}=0 .
\end{gathered}
$$

Eqs. (3), (6) and (8) results in a system of sixteen homogeneous equations in constants ${ }_{j} C_{1},{ }_{j} C_{2},{ }_{j} C_{3},{ }_{j} C_{4},{ }_{j} C_{5},{ }_{j} C_{6},{ }_{j} C_{7}$ and $C_{j}(j=1,2)$ such a homogeneous system has non-trivial solution only if the determinant of the coefficients of the unknowns vanishes identically. Thus by eliminating the constants, the frequency equation of flexural vibrations for poroelastic composite hollow cylinder for a pervious surface is

$$
\left|C_{i j}\right|=0 \text { for } i, j=1,2, \ldots .16
$$

where

$$
\begin{aligned}
& C_{1 j}={ }_{1} M_{1 j}\left(r_{1}\right) ; \quad \mathrm{j}=1,2, \ldots 8, \quad C_{1 j}=0 ; \quad \mathrm{j}=9,10, \ldots 16, \\
& C_{2 j}={ }_{1} M_{2 j}\left(r_{1}\right) ; \quad \mathrm{j}=1,2, \ldots 8, \quad C_{2 j}=0 ; \quad \mathrm{j}=9,10, \ldots 16 \text {, } \\
& C_{3 j}={ }_{1} M_{3 j}\left(r_{1}\right) ; \quad \mathrm{j}=1,2, \ldots 8, \quad C_{3 j}=0 ; \quad \mathrm{j}=9,10, \ldots 16 \text {, } \\
& C_{4 j}={ }_{1} M_{4 j}\left(r_{1}\right) ; \quad \mathrm{j}=1,2,3,4, \quad C_{4 j}=0 ; \quad \mathrm{j}=5,6, \ldots 16 \text {, } \\
& C_{5 j}={ }_{1} M_{1 j}(a) ; \quad \mathrm{j}=1,2, \ldots 8, \quad C_{5 j}={ }_{2} M_{1, j-8}(a) ; \mathrm{j}=9,10, \ldots 16 \text {, } \\
& C_{6 j}={ }_{1} M_{2 j}(a) ; \quad \mathrm{j}=1,2, \ldots 8, \quad C_{6 j}={ }_{2} M_{2, j-8}(a) ; \mathrm{j}=9,10, \ldots 16 \text {, } \\
& C_{7 j}={ }_{1} M_{3 j}(a) ; \quad \mathrm{j}=1,2, \ldots 8, \quad C_{7 j}={ }_{2} M_{3, j-8}(a) ; \mathrm{j}=9,8, \ldots 16 \text {, } \\
& C_{8 j}={ }_{1} M_{4 j}(a) ; \quad \mathrm{j}=1,2,3,4, \quad C_{8 j}=0 ; \quad \mathrm{j}=5,6, \ldots 16 \text {, } \\
& C_{9 j}=0 \quad \mathrm{j}=1,2, \ldots 8,13, \ldots 16, \quad C_{9 j}={ }_{2} M_{4, j-8}(a) ; \mathrm{j}=9, \ldots \ldots 12 \text {, } \\
& C_{10 j}={ }_{1} M_{5 j}(a) ; \quad \mathrm{j}=1,2, \ldots 8, \quad C_{10 j}={ }_{2} M_{5, j-8}(a) ; \mathrm{j}=9,10, \ldots 16, \\
& C_{11 j}={ }_{1} M_{6 j}(a) ; \quad \mathrm{j}=1,2, \ldots 8, \quad C_{11 j}={ }_{2} M_{6, j-8}(a) ; \mathrm{j}=9,10, \ldots 16 \text {, } \\
& C_{12 j}={ }_{1} M_{7 j}(a) ; \quad \mathrm{j}=1,2, \ldots 8, \quad C_{12 j}={ }_{2} M_{7, j-8}(a) ; \mathrm{j}=9,10, \ldots 16 \text {, } \\
& C_{13, j}=0 ; \quad \mathrm{j}=1,2, \ldots 8, \quad C_{13, j}={ }_{2} M_{1, j-8}\left(r_{2}\right) ; \mathrm{j}=9,10, \ldots 16 \text {, } \\
& C_{14, j}=0 ; \quad \mathrm{j}=1,2, \ldots 8, \quad C_{14, j}={ }_{2} M_{2, j-8}\left(r_{2}\right) ; \mathrm{j}=9,10, \ldots 16 \text {, } \\
& C_{15, j}=0 ; \quad \mathrm{j}=1,2, \ldots 8, \quad C_{15, j}={ }_{2} M_{3, j-8}\left(r_{2}\right) ; \mathrm{j}=9,10, \ldots 16 \text {, } \\
& C_{16, j}=0 ; \mathrm{j}=1,2, \ldots 8,13, \ldots 16, \quad C_{16, j}={ }_{2} M_{4, j-8}\left(r_{2}\right) ; \mathrm{j}=9, \ldots . .12 \text {. }
\end{aligned}
$$

In case of an impervious surface, equations (3), (6) and (10) gives the frequency equation as

$$
\left|D_{i j}\right|=0 \text { for } \mathrm{i}, \mathrm{j}=1,2, \ldots .16 \text {, }
$$

where 


$$
\begin{array}{llll}
D_{1 j}={ }_{1} N_{1 j}\left(r_{1}\right) ; & \mathrm{j}=1,2, \ldots 8, & D_{1 j}=0 ; & \mathrm{j}=9,10, \ldots 16, \\
D_{2 j}={ }_{1} N_{2 j}\left(r_{1}\right) ; & \mathrm{j}=1,2, \ldots 8, & D_{2 j}=0 ; & \mathrm{j}=9,10, \ldots 16, \\
D_{3 j}={ }_{1} N_{3 j}\left(r_{1}\right) ; & \mathrm{j}=1,2, \ldots 8, & D_{3 j}=0 ; & \mathrm{j}=9,10, \ldots 16, \\
D_{4 j}={ }_{1} N_{4 j}\left(r_{1}\right) ; & \mathrm{j}=1,2,3,4, & D_{4 j}=0 ; & \mathrm{j}=5,6, \ldots 16, \\
D_{5 j}={ }_{1} N_{1 j}(a) ; & \mathrm{j}=1,2, \ldots 8, & D_{5 j}={ }_{2} N_{1, j-8}(a) ; \mathrm{j}=9,10, \ldots 16, \\
D_{6 j}={ }_{1} N_{2 j}(a) ; & \mathrm{j}=1,2, \ldots 8, & D_{6 j}={ }_{2} N_{2, j-8}(a) ; \mathrm{j}=9,10, \ldots 16, \\
D_{7 j}={ }_{1} N_{3 j}(a) ; & \mathrm{j}=1,2, \ldots 8, & D_{7 j}={ }_{2} N_{3, j-8}(a) ; \mathrm{j}=9,8, \ldots 16, \\
D_{8 j}={ }_{1} N_{4 j}(a) ; & \mathrm{j}=1,2,3,4, & D_{8 j}=0 ; & \mathrm{j}=5,6, \ldots 16, \\
D_{9 j}=0 \quad \mathrm{j}=1,2, \ldots 8,13, \ldots 16, & D_{9 j}={ }_{2} N_{4, j-8}(a) ; \mathrm{j}=9, \ldots .12, \\
D_{10 j}={ }_{1} N_{5 j}(a) ; & \mathrm{j}=1,2, \ldots 8, & D_{10 j}={ }_{2} N_{5, j-8}(a) ; \quad \mathrm{j}=9,10, \ldots 16, \\
D_{11 j}={ }_{1} N_{6 j}(a) ; & \mathrm{j}=1,2, \ldots 8, & D_{11 j}={ }_{2} N_{6, j-8}(a) ; \mathrm{j}=9,10, \ldots 16, \\
D_{12 j}={ }_{1} N_{7 j}(a) ; & \mathrm{j}=1,2, \ldots 8, & D_{12 j}={ }_{2} N_{7, j-8}(a) ; \mathrm{j}=9,10, \ldots 16, \\
D_{13, j}=0 ; & \mathrm{j}=1,2, \ldots 8, & D_{13, j}={ }_{2} N_{1, j-8}\left(r_{2}\right) ; \mathrm{j}=9,10, \ldots 16, \\
D_{14, j}=0 ; & \mathrm{j}=1,2, \ldots 8, & D_{14, j}={ }_{2} N_{2, j-8}\left(r_{2}\right) ; \mathrm{j}=9,10, \ldots 16, \\
D_{15, j}=0 ; & \mathrm{j}=1,2, \ldots 8, & D_{15, j}={ }_{2} N_{3, j-8}\left(r_{2}\right) ; \mathrm{j}=9,10, \ldots 16, \\
D_{16, j}=0 ; & \mathrm{j}=1,2, \ldots 8,13, \ldots 16, & D_{16, j}={ }_{2} N_{4, j-8}\left(r_{2}\right) ; \mathrm{j}=9, \ldots .12 .
\end{array}
$$

and

${ }_{1} N_{l m}(r)={ }_{1} M_{l m}(r)$ for $l=1,2,3,5,6,7,8 \quad m=1$ to 8 ,

${ }_{2} N_{l m}(r)={ }_{2} M_{l m}(r)$ for $l=1,2,3,5,6,7,8 \quad m=1$ to 8 ,

${ }_{j} N_{4 m}(r)$ for $\mathrm{m}=1,2,3,4$ and ${ }_{j} M_{l m}(r)$ for all $l, m$ are defined in equations (3) and (7).

\section{Motions having infinite wavelength}

When the wavelength is infinite or the wavenumber is zero, the frequency equation (10) of poroelastic composite hollow cylinder for a pervious surface reduces to

with

$$
A_{1} A_{2}=0
$$

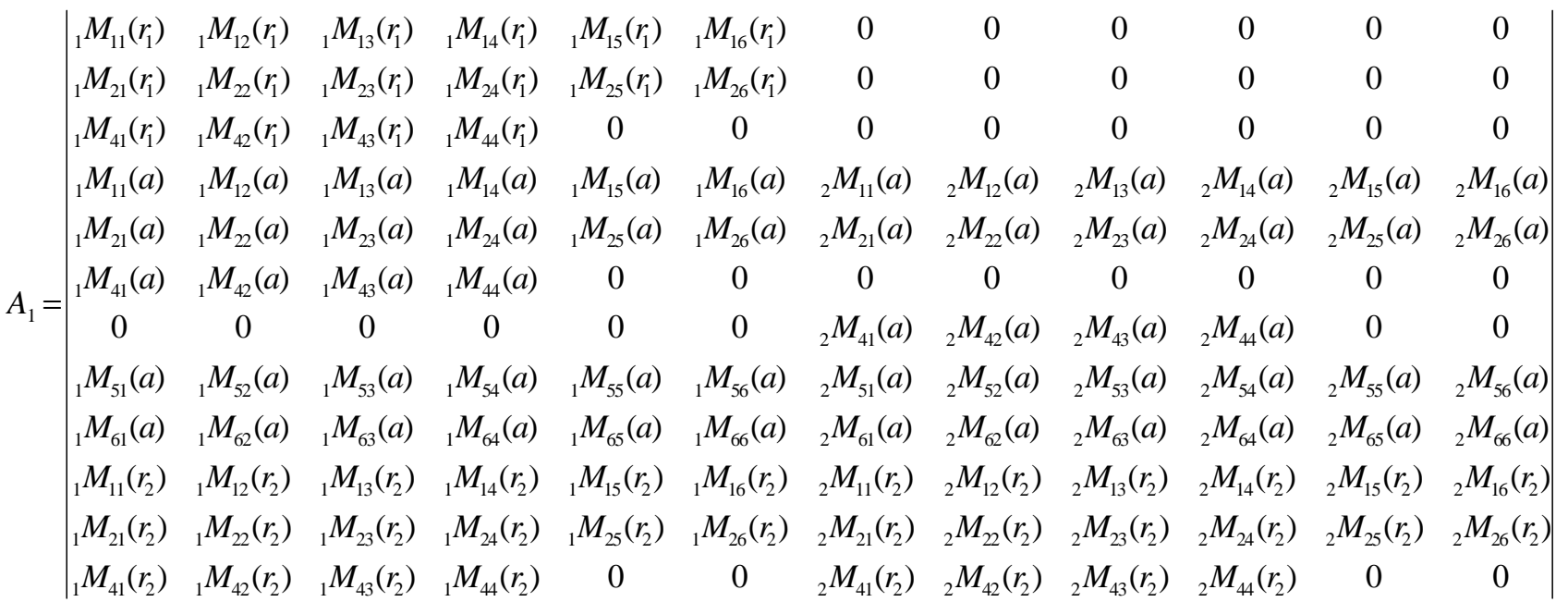


$A_{2}=\left|\begin{array}{cccc}{ }_{1} M_{37}\left(r_{1}\right) & { }_{1} M_{38}\left(r_{1}\right) & 0 & 0 \\ { }_{1} M_{37}(a) & { }_{1} M_{38}(a) & { }_{2} M_{37}(a) & { }_{2} M_{38}(a) \\ { }_{1} M_{77}(a) & { }_{1} M_{78}(a) & { }_{2} M_{77}(a) & { }_{2} M_{78}(a) \\ 0 & 0 & { }_{2} M_{37}\left(r_{2}\right) & { }_{2} M_{38}\left(r_{2}\right)\end{array}\right|$

where the elements ${ }_{j} M_{l m}(r)$ are defined in eqs. (4) and (7) for $k=0$. From eq. (14) it is clear that either $A_{1}=0$ or $A_{2}=0$. The equation

$$
A_{1}=0
$$

is the frequency equation of plane-strain vibrations of a poroelastic composite hollow cylinder for infinite wavelength in case of a pervious surface. The frequency equation

$$
A_{2}=0
$$

involves only shear wave velocity, hence it is the frequency equation of longitudinal shear vibrations of a poroelastic composite hollow cylinder for infinite wavelength in case of a pervious surface. Eq. (14) shows that the plane-strain vibrations and longitudinal shear vibrations of poroelastic composite hollow cylinder for a pervious surface are uncoupled when wavelength is infinite.

Similarly, the frequency equation (12) of vibrations in poroelastic composite hollow cylinder for an impervious surface reduces to

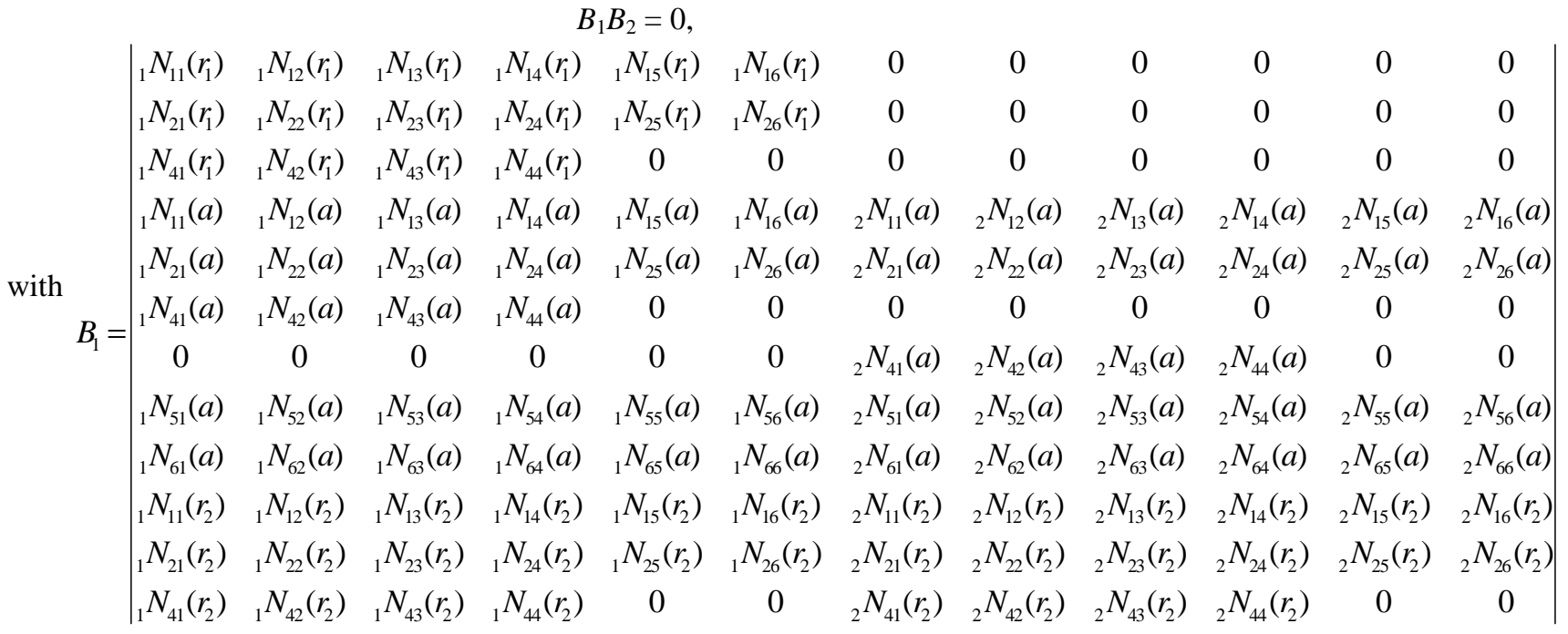

$$
\begin{aligned}
& B_{2}=\left|\begin{array}{cccc}
{ }_{1} N_{37}\left(r_{1}\right) & { }_{1} N_{38}\left(r_{1}\right) & 0 & 0 \\
{ }_{1} N_{37}(a) & { }_{1} N_{38}(a) & { }_{2} N_{37}(a) & { }_{2} N_{38}(a) \\
{ }_{1} N_{77}(a) & { }_{1} N_{78}(a) & { }_{2} N_{77}(a) & { }_{2} N_{78}(a) \\
0 & 0 & { }_{2} N_{37}\left(r_{2}\right) & { }_{2} N_{38}\left(r_{2}\right)
\end{array}\right|
\end{aligned}
$$

where the elements ${ }_{j} N_{l m}(r)$ are defined in eq. (7) and (13) and are calculated for $k=0$.

From eq. (18) it is clear that $B_{1}=0$ or $B_{2}=0$. Equation

$$
B_{1}=0
$$

is the frequency equation of plane-strain vibrations of poroelastic composite hollow cylinder for an impervious surface when wavelength is infinite, whereas the equation

$$
B_{2}=0
$$

is the frequency equation of longitudinal shear vibrations of poroelastic composite hollow cylinder for an impervious surface when wavelength is infinite. Eq. (18) shows that the plane-strain vibrations and longitudinal shear vibrations of poroelastic 
composite hollow cylinder for an impervious surface are uncoupled. Also, we see that the equations $A_{2}=0$ and $B_{2}=0$ are same, hence the frequency equation of longitudinal shear vibrations of poroelastic composite hollow cylinder is independent of nature of surface for infinite wavelength.

\section{Particular cases}

Under suitable boundary conditions the poroelastic composite hollow cylinder reduces to the following particular cases 4.1 Poroelastic composite hollow cylinder with rigid casing,

4.2 Poroelastic composite bore.

\subsection{Poroelastic composite hollow cylinder with rigid casing}

When shear modulus of the casing is larger than that of core, we can assume that casing is perfectly rigid. Letting the shear modulus of the casing approaches to infinity i.e., $N^{(2)} \rightarrow \infty$, then the shear wave velocity of casing approaches to infinity and hence $\xi_{3}^{(2)} \rightarrow 0$. Under this limiting condition, the frequency equation (10) of vibrations of poroelastic composite hollow cylinder for a pervious surface reduces to

$$
\begin{aligned}
& C_{1} C_{2}=0 \\
& \text { with } \quad C_{1}=\left|\begin{array}{llllllll}
{ }_{1} M_{11}\left(r_{1}\right) & { }_{1} M_{12}\left(r_{1}\right) & { }_{1} M_{13}\left(r_{1}\right) & { }_{1} M_{14}\left(r_{1}\right) & { }_{1} M_{15}\left(r_{1}\right) & { }_{1} M_{16}\left(r_{1}\right) & { }_{1} M_{17}\left(r_{1}\right) & { }_{1} M_{18}\left(r_{1}\right) \\
{ }_{1} M_{21}\left(r_{1}\right) & { }_{1} M_{22}\left(r_{1}\right) & { }_{1} M_{23}\left(r_{1}\right) & { }_{1} M_{24}\left(r_{1}\right) & { }_{1} M_{25}\left(r_{1}\right) & { }_{1} M_{26}\left(r_{1}\right) & { }_{1} M_{27}\left(r_{1}\right) & { }_{1} M_{28}\left(r_{1}\right) \\
{ }_{1} M_{31}\left(r_{1}\right) & { }_{1} M_{32}\left(r_{1}\right) & { }_{1} M_{33}\left(r_{1}\right) & { }_{1} M_{34}\left(r_{1}\right) & { }_{1} M_{35}\left(r_{1}\right) & { }_{1} M_{36}\left(r_{1}\right) & { }_{1} M_{37}\left(r_{1}\right) & { }_{1} M_{38}\left(r_{1}\right) \\
{ }_{1} M_{41}\left(r_{1}\right) & { }_{1} M_{42}\left(r_{1}\right) & { }_{1} M_{43}\left(r_{1}\right) & { }_{1} M_{44}\left(r_{1}\right) & 0 & 0 & 0 & 0 \\
{ }_{1} M_{41}(a) & { }_{1} M_{42}(a) & { }_{1} M_{43}(a) & { }_{1} M_{44}(a) & 0 & 0 & 0 & 0 \\
{ }_{1} M_{51}(a) & { }_{1} M_{52}(a) & { }_{1} M_{53}(a) & { }_{1} M_{54}(a) & { }_{1} M_{55}(a) & { }_{1} M_{56}(a) & { }_{1} M_{57}(a) & { }_{1} M_{58}(a) \\
{ }_{1} M_{61}(a) & { }_{1} M_{62}(a) & { }_{1} M_{63}(a) & { }_{1} M_{64}(a) & { }_{1} M_{65}(a) & { }_{1} M_{66}(a) & { }_{1} M_{67}(a) & { }_{1} M_{68}(a) \\
{ }_{1} M_{71}(a) & { }_{1} M_{72}(a) & { }_{1} M_{73}(a) & { }_{1} M_{74}(a) & 0 & 0 & { }_{1} M_{77}(a) & { }_{1} M_{78}(a)
\end{array}\right| \\
& C_{2}=\left|\begin{array}{llllllll}
{ }_{2} A_{11}(\mathrm{a}) & { }_{2} A_{12}(\mathrm{a}) & { }_{2} A_{13}(\mathrm{a}) & { }_{2} A_{14}(\mathrm{a}) & { }_{2} A_{15}(\mathrm{a}) & { }_{2} A_{16}(\mathrm{a}) & { }_{2} A_{17}(\mathrm{a}) & { }_{2} A_{18}(\mathrm{a}) \\
{ }_{2} A_{21}(\mathrm{a}) & { }_{2} A_{22}(\mathrm{a}) & { }_{2} A_{23}(\mathrm{a}) & { }_{2} A_{24}(\mathrm{a}) & { }_{2} A_{25}(\mathrm{a}) & { }_{2} A_{26}(\mathrm{a}) & { }_{2} A_{27}(\mathrm{a}) & { }_{2} A_{28}(\mathrm{a}) \\
{ }_{2} A_{31}(\mathrm{a}) & { }_{2} A_{32}(\mathrm{a}) & { }_{2} A_{33}(\mathrm{a}) & { }_{2} A_{34}(\mathrm{a}) & { }_{2} A_{35}(\mathrm{a}) & { }_{2} A_{36}(\mathrm{a}) & { }_{2} A_{37}(\mathrm{a}) & { }_{2} A_{38}(\mathrm{a}) \\
{ }_{2} A_{41}(\mathrm{a}) & { }_{2} A_{42}(\mathrm{a}) & { }_{2} A_{43}(\mathrm{a}) & { }_{2} A_{44}(\mathrm{a}) & { }_{2} A_{45}(\mathrm{a}) & { }_{2} A_{46}(\mathrm{a}) & { }_{2} A_{47}(\mathrm{a}) & { }_{2} A_{48}(\mathrm{a}) \\
{ }_{2} A_{11}\left(\mathrm{r}_{2}\right) & { }_{2} A_{12}\left(\mathrm{r}_{2}\right) & { }_{2} A_{13}\left(\mathrm{r}_{2}\right) & { }_{2} A_{14}\left(\mathrm{r}_{2}\right) & { }_{2} A_{15}\left(\mathrm{r}_{2}\right) & { }_{2} A_{16}\left(\mathrm{r}_{2}\right) & { }_{2} A_{17}\left(\mathrm{r}_{2}\right) & { }_{2} A_{18}\left(\mathrm{r}_{2}\right) \\
{ }_{2} A_{21}\left(\mathrm{r}_{2}\right) & { }_{2} A_{22}\left(\mathrm{r}_{2}\right) & { }_{2} A_{23}\left(\mathrm{r}_{2}\right) & { }_{2} A_{24}\left(\mathrm{r}_{2}\right) & { }_{2} A_{25}\left(\mathrm{r}_{2}\right) & { }_{2} A_{26}\left(\mathrm{r}_{2}\right) & { }_{2} A_{27}\left(\mathrm{r}_{2}\right) & { }_{2} A_{28}\left(\mathrm{r}_{2}\right) \\
{ }_{2} A_{31}\left(\mathrm{r}_{2}\right) & { }_{2} A_{32}\left(\mathrm{r}_{2}\right) & { }_{2} A_{33}\left(\mathrm{r}_{2}\right) & { }_{2} A_{34}\left(\mathrm{r}_{2}\right) & { }_{2} A_{35}\left(\mathrm{r}_{2}\right) & { }_{2} A_{36}\left(\mathrm{r}_{2}\right) & { }_{2} A_{37}\left(\mathrm{r}_{2}\right) & { }_{2} A_{38}\left(\mathrm{r}_{2}\right) \\
{ }_{2} A_{41}\left(\mathrm{r}_{2}\right) & { }_{2} A_{42}\left(\mathrm{r}_{2}\right) & { }_{2} A_{43}\left(\mathrm{r}_{2}\right) & { }_{2} A_{44}\left(\mathrm{r}_{2}\right) & { }_{2} A_{45}\left(\mathrm{r}_{2}\right) & { }_{2} A_{46}\left(\mathrm{r}_{2}\right) & { }_{2} A_{47}\left(\mathrm{r}_{2}\right) & { }_{2} A_{48}\left(\mathrm{r}_{2}\right)
\end{array}\right|
\end{aligned}
$$

where

$$
\begin{aligned}
& { }_{2} A_{11}(r)=\left\{\left({ }_{2} Q+{ }_{2} R\right)_{2} \alpha_{1}^{2} k^{2}+\left({ }_{2} Q+{ }_{2} R\right)_{2} \alpha_{1}^{2}-2{ }_{2} \alpha_{1}^{2}\right\} J_{0}\left({ }_{2} \xi_{1} r\right)+\frac{2{ }_{2} \alpha_{1}}{r} J_{1}\left({ }_{2} \alpha_{1} r\right), \\
& { }_{2} A_{12}(r)=\left\{\left({ }_{2} Q+{ }_{2} R\right)_{2} \alpha_{1}^{2} k^{2}+\left({ }_{2} Q+{ }_{2} R\right)_{2} \alpha_{1}^{2}-2{ }_{2} \alpha_{1}^{2}\right\} Y_{0}\left({ }_{2} \xi_{1} r\right)+\frac{2{ }_{2} \alpha_{1}}{r} Y_{1}\left({ }_{2} \alpha_{1} r\right), \\
& { }_{2} A_{13}(r)=\left\{\left({ }_{2} Q+{ }_{2} R\right){ }_{2} \alpha_{2}^{2} k^{2}+\left({ }_{2} Q+{ }_{2} R\right)_{2} \alpha_{2}^{2}-2{ }_{2} \alpha_{2}^{2}\right\} J_{0}\left({ }_{2} \xi_{2} r\right)+\frac{2{ }_{2} \alpha_{2}}{r} J_{1}\left({ }_{2} \alpha_{2} r\right),
\end{aligned}
$$




$$
\begin{aligned}
& { }_{2} A_{14}(r)=\left\{\left({ }_{2} Q+{ }_{2} R\right)_{2} \alpha_{2}^{2} k^{2}+\left({ }_{2} Q+{ }_{2} R\right){ }_{2} \alpha_{2}^{2}-2{ }_{2} \alpha_{2}^{2}\right\} Y_{0}\left({ }_{2} \xi_{2} r\right)+\frac{2{ }_{2} \alpha_{2}}{r} Y_{1}\left({ }_{2} \alpha_{2} r\right), \\
& { }_{2} A_{15}(r)=-2 i k_{2} \alpha_{3} J_{0}\left({ }_{2} \xi_{3} r\right)+\frac{2 i k}{r} J_{1}\left({ }_{2} \xi_{3} r\right) \\
& { }_{2} A_{16}(r)=-2 i k_{2} \alpha_{3} J_{0}\left({ }_{2} \xi_{3} r\right)+\frac{2 i k}{r} Y_{1}\left({ }_{2} \xi_{3} r\right) \\
& { }_{2} A_{21}(r)=\frac{2{ }_{2} \xi_{1}}{r} J_{2}\left({ }_{2} \xi_{1} r\right), \quad{ }_{2} A_{22}(r)=\frac{2 \xi_{2} \xi_{1}}{r} Y_{2}\left({ }_{2} \xi_{1} r\right), \\
& { }_{2} A_{23}(r)=\frac{2 \xi_{2} \xi_{1}}{r} J_{2}\left({ }_{2} \xi_{2} r\right), \quad{ }_{2} A_{24}(r)=\frac{2{ }_{2} \xi_{2}}{r} J_{2}\left({ }_{2} \xi_{2} r\right), \\
& { }_{2} A_{25}(r)={ }_{2} \xi_{1}^{2} J_{1}\left({ }_{2} \xi_{3} r\right)-\frac{2 \xi_{2} \xi_{3}}{r} J_{2}\left({ }_{2} \xi_{3} r\right), \\
& { }_{2} A_{26}(r)={ }_{2} \xi_{1}^{2} Y_{1}\left({ }_{2} \xi_{3} r\right)-\frac{2{ }_{2} \xi_{3}}{r} Y_{2}\left({ }_{2} \xi_{3} r\right), \\
& { }_{2} A_{27}(r)=i k_{2} \xi_{3} J_{1}\left({ }_{2} \xi_{3} r\right) \\
& { }_{2} A_{28}(r)=i k_{2} \xi_{3} Y_{1}\left({ }_{2} \xi_{3} r\right) \text {, } \\
& { }_{2} A_{41}(r)={ }_{2} R{ }_{2} \alpha_{1}^{2}\left(k^{2}+{ }_{2} \xi_{1}^{2}\right) J_{1}\left({ }_{2} \xi_{1} r\right), \quad{ }_{2} A_{42}(r)={ }_{2} R{ }_{2} \alpha_{1}^{2}\left(k^{2}+{ }_{2} \xi_{1}^{2}\right) Y_{1}\left({ }_{2} \xi_{1} r\right) \text {, } \\
& { }_{2} A_{43}(r)={ }_{2} R{ }_{2} \alpha_{2}^{2}\left(k^{2}+{ }_{2} \xi_{1}^{2}\right) J_{1}\left({ }_{2} \xi_{2} r\right), \quad{ }_{2} A_{44}(r)={ }_{2} R{ }_{2} \alpha_{2}^{2}\left(k^{2}+{ }_{2} \xi_{2}^{2}\right) Y_{1}\left({ }_{2} \xi_{2} r\right), \\
& \text { and } \\
& { }_{2} \alpha_{i}^{2}=\frac{-2{ }_{2} V_{i}^{-2}{ }_{2} R}{{ }_{2} R_{2} k_{12}-{ }_{2} Q_{2} k_{22}} \text { for } i=1,2 \text { and } 3 \text {. }
\end{aligned}
$$

From eq. (22) it is clear that the physical parameters in the determinants $C_{1}, C_{2}$ are, respectively, related to core and casing. Hence, the vibrations of poroelastic composite hollow cylinder related to core and casing for a pervious surface are uncoupled when the solid in casing is rigid, also we obtain $C_{1}=0$ or $C_{2}=0$. The equation

$$
C_{1}=0
$$

represents the frequency equation of vibrations of poroelastic core for a pervious surface when it is clamped along its outer surface, whereas the equation

$$
C_{2}=0
$$

represents the frequency equation of vibrations of hollow rigid casing for a pervious surface when the boundaries are free from stress.

In a similar way, when the solid in casing is rigid, the frequency eq. (12) of vibrations of poroelastic composite hollow cylinder for an impervious surface reduces to

with

$$
D_{1} D_{2}=0 \text {, }
$$




$$
D_{1}=\left|\begin{array}{llllcccc}
{ }_{1} N_{11}\left(r_{1}\right) & { }_{1} N_{12}\left(r_{1}\right) & { }_{1} N_{13}\left(r_{1}\right) & { }_{1} N_{14}\left(r_{1}\right) & { }_{1} N_{15}\left(r_{1}\right) & { }_{1} N_{16}\left(r_{1}\right) & { }_{1} N_{17}\left(r_{1}\right) & { }_{1} N_{18}\left(r_{1}\right) \\
{ }_{1} N_{21}\left(r_{1}\right) & { }_{1} N_{22}\left(r_{1}\right) & { }_{1} N_{23}\left(r_{1}\right) & { }_{1} N_{24}\left(r_{1}\right) & { }_{1} N_{25}\left(r_{1}\right) & { }_{1} N_{26}\left(r_{1}\right) & { }_{1} N_{27}\left(r_{1}\right) & { }_{1} N_{28}\left(r_{1}\right) \\
{ }_{1} N_{31}\left(r_{1}\right) & { }_{1} N_{32}\left(r_{1}\right) & { }_{1} N_{33}\left(r_{1}\right) & { }_{1} N_{34}\left(r_{1}\right) & { }_{1} N_{35}\left(r_{1}\right) & { }_{1} N_{36}\left(r_{1}\right) & { }_{1} N_{37}\left(r_{1}\right) & { }_{1} N_{38}\left(r_{1}\right) \\
{ }_{1} N_{41}\left(r_{1}\right) & { }_{1} N_{42}\left(r_{1}\right) & { }_{1} N_{43}\left(r_{1}\right) & { }_{1} N_{44}\left(r_{1}\right) & 0 & 0 & 0 & 0 \\
{ }_{1} N_{41}(a) & { }_{1} N_{42}(a) & { }_{1} N_{43}(a) & { }_{1} N_{44}(a) & 0 & 0 & 0 & 0 \\
{ }_{1} N_{51}(a) & { }_{1} N_{52}(a) & { }_{1} N_{53}(a) & { }_{1} N_{54}(a) & { }_{1} N_{55}(a) & { }_{1} N_{56}(a) & { }_{1} N_{57}(a) & { }_{1} N_{58}(a) \\
{ }_{1} N_{61}(a) & { }_{1} N_{62}(a) & { }_{1} N_{63}(a) & { }_{1} N_{64}(a) & { }_{1} N_{65}(a) & { }_{1} N_{66}(a) & { }_{1} N_{67}(a) & { }_{1} N_{68}(a) \\
{ }_{1} N_{71}(a) & { }_{1} N_{72}(a) & { }_{1} N_{73}(a) & { }_{1} N_{74}(a) & 0 & 0 & { }_{1} N_{77}(a) & { }_{1} N_{78}(a)
\end{array}\right|
$$

and

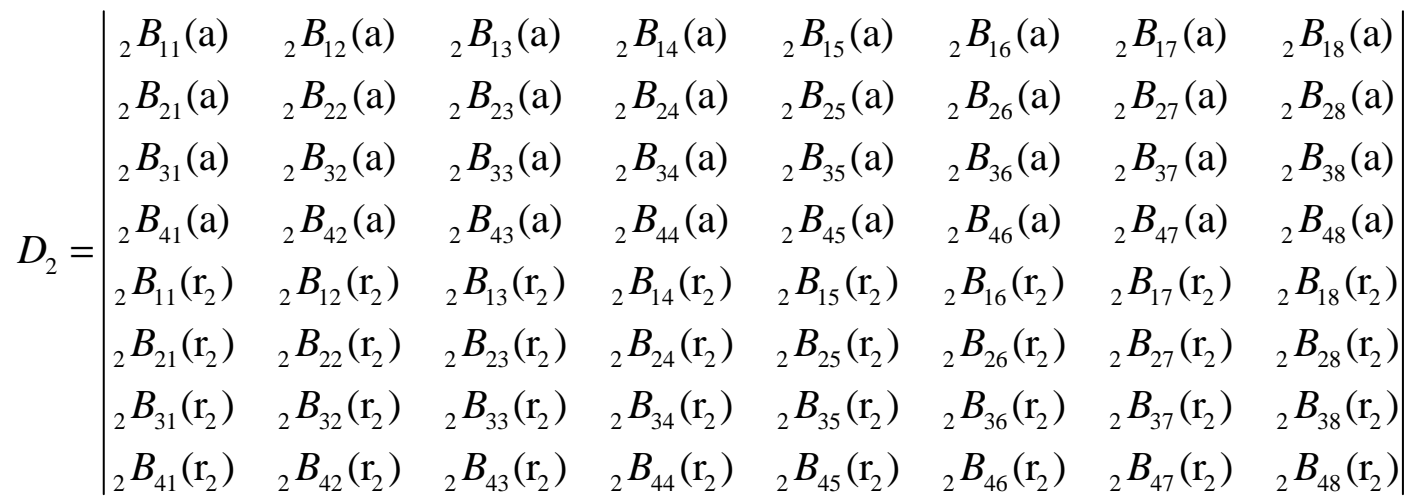

where

$$
\begin{aligned}
& { }_{2} B_{1 m}(r)={ }_{2} A_{1 m}(r) \text { for } m=1,2, \ldots . .8 \text {, } \\
& { }_{2} B_{2 m}(r)={ }_{2} A_{2 m}(r) \text { for } m=1,2, \ldots . .8 \text {, } \\
& { }_{2} B_{3 m}(r)={ }_{2} A_{3 m}(r) \text { for } m=1,2, \ldots . .8 \text {, } \\
& { }_{2} B_{41}(r)=\frac{\left({ }_{2} R_{2} \alpha_{1}^{2}-{ }_{2} Q\right)\left(k^{2}+{ }_{2} \xi_{1}^{2}\right)}{r} J_{1}\left({ }_{2} \xi_{1} r\right)-{ }_{2} \xi_{1}\left({ }_{2} R_{2} \alpha_{1}^{2}-{ }_{2} Q\right)\left(k^{2}+{ }_{2} \xi_{1}^{2}\right) J_{2}\left({ }_{2} \xi_{1} r\right), \\
& { }_{2} B_{42}(r)=\frac{\left({ }_{2} R_{2} \alpha_{1}^{2}-{ }_{2} Q\right)\left(k^{2}+{ }_{2} \xi_{1}^{2}\right)}{r} Y_{1}\left({ }_{2} \xi_{1} r\right)-{ }_{2} \xi_{1}\left({ }_{2} R{ }_{2} \alpha_{1}^{2}-{ }_{2} Q\right)\left(k^{2}+{ }_{2} \xi_{1}^{2}\right) Y_{2}\left({ }_{2} \xi_{1} r\right), \\
& { }_{2} B_{43}(r)=\frac{\left({ }_{2} R_{2} \alpha_{2}{ }^{2}-{ }_{2} Q\right)\left(k^{2}+{ }_{2} \xi_{2}{ }^{2}\right)}{r} J_{1}\left({ }_{2} \xi_{2} r\right)-{ }_{2} \xi_{2}\left({ }_{2} R_{2} \alpha_{2}{ }^{2}-{ }_{2} Q\right)\left(k^{2}+{ }_{2} \xi_{2}{ }^{2}\right) J_{2}\left({ }_{2} \xi_{2} r\right), \\
& { }_{2} B_{44}(r)=\frac{\left({ }_{2} R_{2} \alpha_{2}{ }^{2}-{ }_{2} Q\right)\left(k^{2}+{ }_{2} \xi_{2}{ }^{2}\right)}{r} Y_{1}\left({ }_{2} \xi_{2} r\right)-{ }_{2} \xi_{2}\left({ }_{2} R_{2} \alpha_{2}{ }^{2}-{ }_{2} Q\right)\left(k^{2}+{ }_{2} \xi_{2}{ }^{2}\right) Y_{2}\left({ }_{2} \xi_{2} r\right) \text {. }
\end{aligned}
$$

and ${ }_{j} N_{l m}(r)$ are defined in eqs.(7) and (13) and ${ }_{2} A_{l m}(r)$ are defined in eq. (24).

As in the case of a pervious surface, the vibrations of poroelastic composite hollow cylinder related to core and casing for an impervious surface are uncoupled when the solid in casing is rigid. From eq. (27) it is clear that $D_{1}=0$ or $D_{2}=0$. The equation

$$
D_{1}=0 \text {, }
$$

represents the frequency equation of vibrations poroelastic core for an impervious surface when it is clamped along its outer surface, whereas the equation 


$$
D_{2}=0 \text {, }
$$

represents the frequency equation of vibrations of hollow rigid casing for an impervious surface when the boundaries are free from stress.

\subsection{Poroelastic composite bore}

When the outer radius $r_{2}$ of casing tends to, the frequency equation (10) of poroelastic composite hollow cylinder for a pervious surface reduces to

$$
E_{1}=0
$$

where

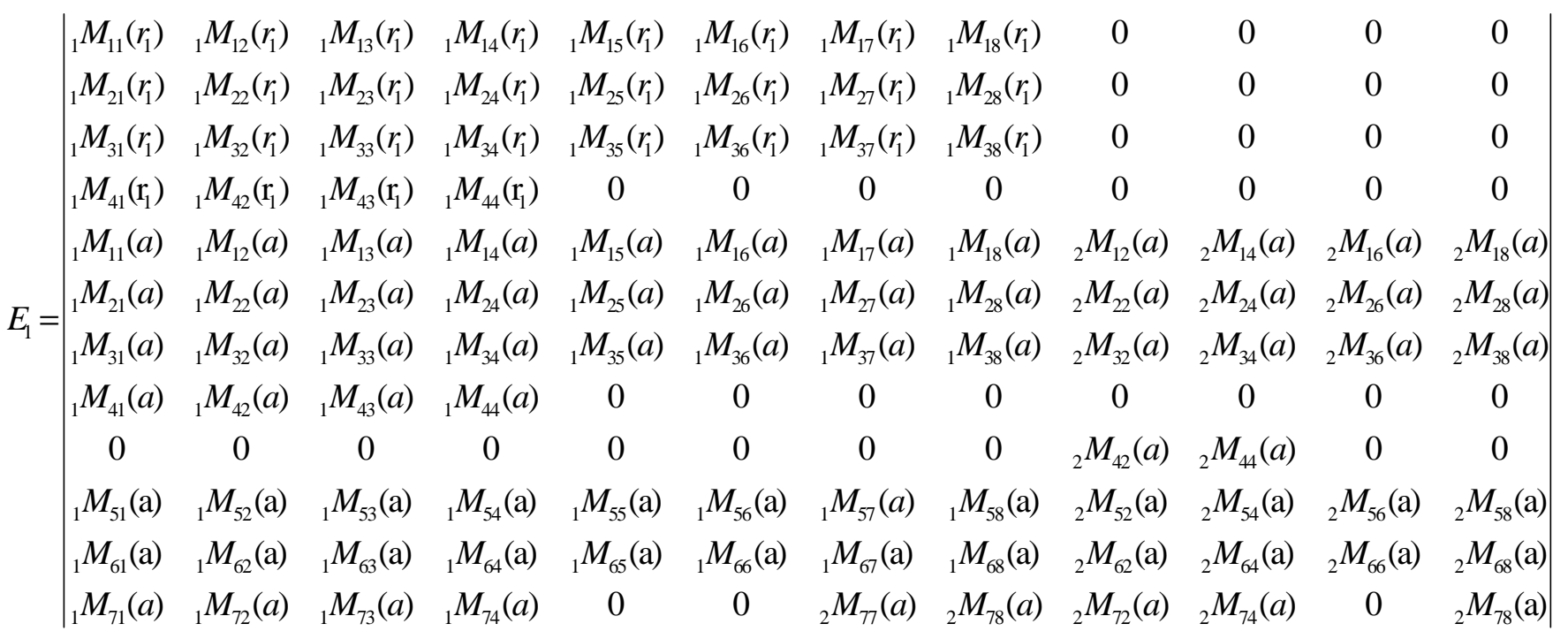

where the elements ${ }_{j} M_{\mathrm{lm}}$ are defined in eq. (8).

Eq. (32) is the frequency equation of flexural vibrations in poroelastic composite bore for a pervious surface. obtained as

Similarly, the frequency equation of flexural vibrations in poroelastic composite bore for an impervious surface can be

$$
F_{1}=0
$$




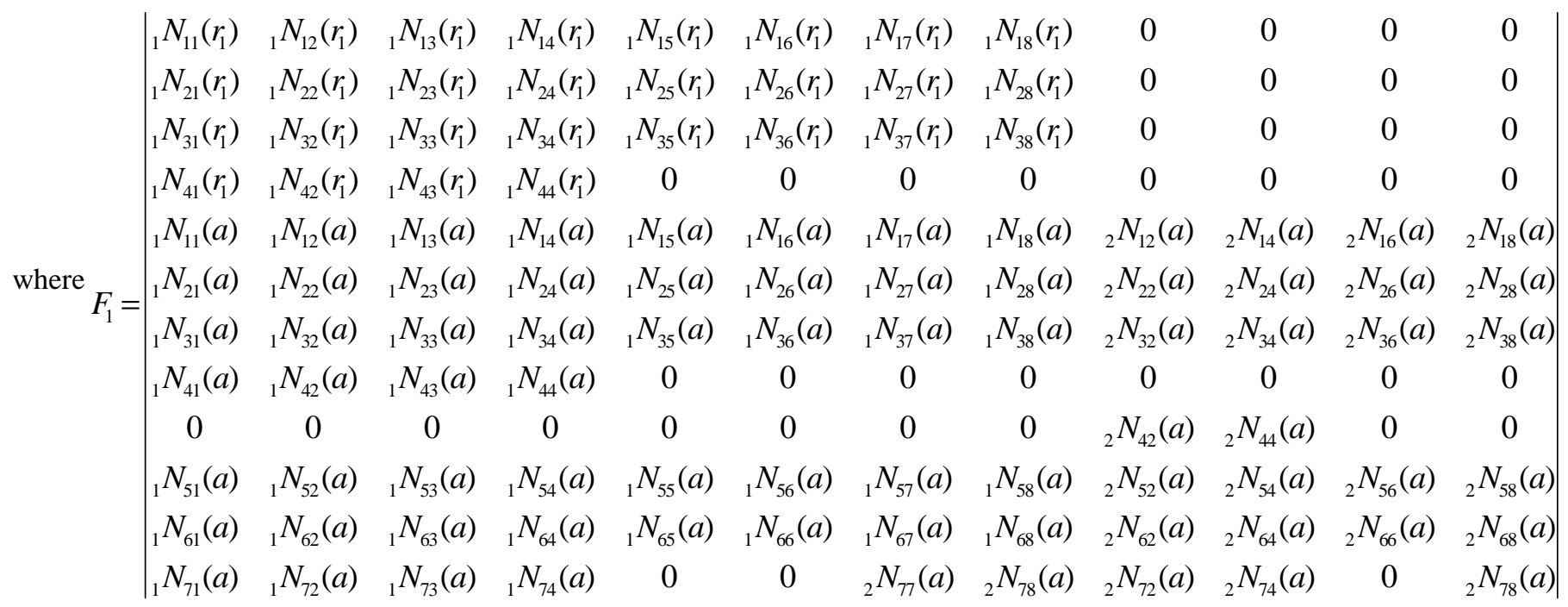

where the elements ${ }_{j} N_{\mathrm{lm}}$ are defined in eqs. (7) and (13).

For infinite wavelength, the frequency equation $E_{1}=0$ of flexural vibrations of poroelastic composite bore for a pervious surface reduces to

$$
E_{2} E_{3}=0
$$

with

$$
E_{2}=\left|\begin{array}{ccccccccc}
{ }_{1} M_{11}\left(r_{1}\right) & { }_{1} M_{12}\left(r_{1}\right) & { }_{1} M_{13}\left(r_{1}\right) & { }_{1} M_{14}\left(r_{1}\right) & { }_{1} M_{15}\left(r_{1}\right) & { }_{1} M_{16}\left(r_{1}\right) & 0 & 0 & 0 \\
{ }_{1} M_{21}\left(r_{1}\right) & { }_{1} M_{22}\left(r_{1}\right) & { }_{1} M_{23}\left(r_{1}\right) & { }_{1} M_{24}\left(r_{1}\right) & { }_{1} M_{25}\left(r_{1}\right) & { }_{1} M_{26}\left(r_{1}\right) & 0 & 0 & 0 \\
{ }_{1} M_{41}\left(r_{1}\right) & { }_{1} M_{42}\left(r_{1}\right) & { }_{1} M_{43}\left(r_{1}\right) & { }_{1} M_{44}\left(r_{1}\right) & 0 & 0 & 0 & 0 & 0 \\
{ }_{1} M_{11}(a) & { }_{1} M_{12}(a) & { }_{1} M_{13}(a) & { }_{1} M_{14}(a) & { }_{1} M_{15}(a) & { }_{1} M_{16}(a) & { }_{2} M_{12}(a) & { }_{2} M_{14}(a) & { }_{2} M_{16}(a) \\
{ }_{1} M_{21}(a) & { }_{1} M_{22}(a) & { }_{1} M_{23}(a) & { }_{1} M_{24}(a) & { }_{1} M_{25}(a) & { }_{1} M_{26}(a) & { }_{2} M_{22}(a) & { }_{2} M_{24}(a) & { }_{2} M_{26}(a) \\
{ }_{1} M_{41}(a) & { }_{1} M_{42}(a) & { }_{1} M_{43}(a) & { }_{1} M_{44}(a) & 0 & 0 & 0 & 0 & 0 \\
0 & 0 & 0 & 0 & 0 & 0 & { }_{2} M_{42}(a) & { }_{2} M_{44}(a) & 0 \\
{ }_{1} M_{51}(a) & { }_{1} M_{52}(a) & { }_{1} M_{53}(a) & { }_{1} M_{54}(a) & { }_{1} M_{55}(a) & { }_{1} M_{56}(a) & { }_{2} M_{52}(a) & { }_{2} M_{54}(a) & { }_{2} M_{56}(a) \\
{ }_{1} M_{61}(a) & { }_{1} M_{62}(a) & { }_{1} M_{63}(a) & { }_{1} M_{64}(a) & { }_{1} M_{65}(a) & { }_{1} M_{66}(a) & { }_{2} M_{62}(a) & { }_{2} M_{64}(a) & { }_{2} M_{66}(a)
\end{array}\right|
$$

and

$$
E_{3}=\left|\begin{array}{ccc}
{ }_{1} M_{37}\left(r_{1}\right) & { }_{1} M_{38}\left(r_{1}\right) & 0 \\
{ }_{1} M_{37}(a) & { }_{1} M_{37}(a) & { }_{1} M_{37}(a) \\
{ }_{1} M_{77}(a) & { }_{1} M_{78}(a) & { }_{2} M_{78}(a)
\end{array}\right|
$$

where the elements ${ }_{j} M_{\mathrm{lm}}$ are defined in eq. (7) and are evaluated for $k=0$.

From eq. (36), it is clear that either $E_{2}=0$ or $E_{3}=0$. In particular,

$$
E_{2}=0
$$

is the frequency equation of plane-strain vibrations of poroelastic composite bore for a pervious surface, whereas the equation

$$
E_{3}=0 \text {, }
$$

is the frequency equation of longitudinal shear vibrations of poroelastic composite bore for a pervious surface. Eq. (36) shows that the plane-strain vibrations and longitudinal shear vibrations of poroelastic composite bore for a pervious surface are uncoupled. 
In a similar way, for infinite wavelength, the frequency equation $F_{1}=0$ of vibrations of poroelastic composite bore for an impervious surface reduces to

$$
F_{2} F_{3}=0
$$

with

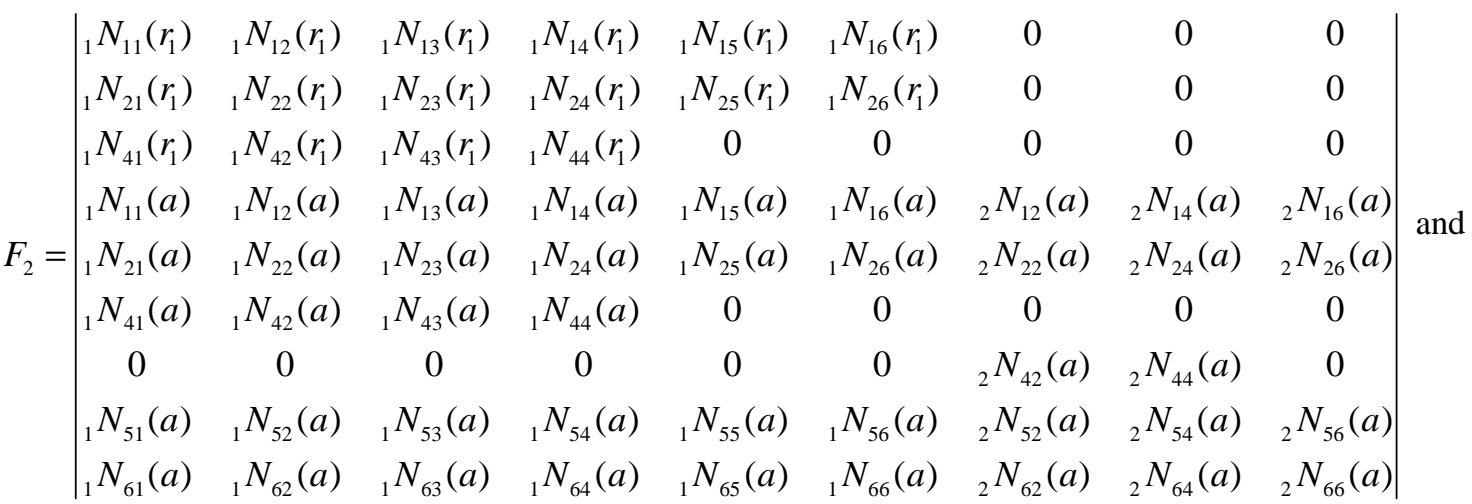

$$
\begin{aligned}
& F_{3}=\left|\begin{array}{ccc}
{ }_{1} N_{37}\left(r_{1}\right) & { }_{1} N_{38}\left(r_{1}\right) & 0 \\
{ }_{1} N_{37}(a) & { }_{1} N_{37}(a) & { }_{1} N_{37}(a) \\
{ }_{1} N_{77}(a) & { }_{1} N_{78}(a) & { }_{2} N_{78}(a)
\end{array}\right|
\end{aligned}
$$

where the elements ${ }_{j} N_{1 \mathrm{~m}}$ are defined in eq. (13) and are evaluated for $k=0$.

From eq. (40), clearly $F_{2}=0$ or $F_{3}=0$. In particular,

$$
F_{2}=0 \text {, }
$$

is the frequency equation of plane-strain vibrations of poroelastic composite bore for an impervious surface.

The equation

$$
F_{3}=0 \text {, }
$$

is the frequency equation of longitudinal shear vibrations of poroelastic composite bore for an impervious surface which is same as equation $E_{3}=0$ using (13), hence the frequency equation of longitudinal shear vibrations of poroelastic composite bore is independent of nature of surface for infinite wavelength. Eq. (40) shows that the plane-strain vibrations and longitudinal vibrations of poroelastic composite bore for an impervious surface are uncoupled for infinite wavelength.

\section{Non-dimensionalization of frequency equation}

The natural frequency will be real when the dissipation coefficient is zero i.e. $b=0$. For the sake of numerical work the dissipation coefficient ' $b$ ' is taken as zero and hence we obtained only real frequency. To analyze the frequency equations of vibrations of poroelastic composite hollow cylinders, it is convenient to introduce the following non-dimensional parameters:

$$
\begin{aligned}
& a_{1}=\frac{{ }_{2} P}{{ }_{1} H}, \quad a_{2}=\frac{{ }_{2} Q}{{ }_{1} H}, \quad a_{3}=\frac{{ }_{2} R}{{ }_{1} H}, \quad a_{4}=\frac{{ }_{2} N}{{ }_{1} H}, \quad d_{1}=\frac{{ }_{2} \rho_{11}}{{ }_{1} \rho}, d_{2}=\frac{{ }_{2} \rho_{12}}{{ }_{1} \rho}, d_{3}=\frac{{ }_{2} \rho_{22}}{{ }_{1} \rho}, \\
& b_{1}=\frac{{ }_{1} P}{{ }_{1} H}, \quad b_{2}=\frac{{ }_{1} Q}{{ }_{1} H}, \quad b_{3}=\frac{{ }_{1} R}{{ }_{1} H}, \quad b_{4}=\frac{1}{{ }_{1} H}, \quad g_{1}=\frac{{ }_{1} \rho_{11}}{{ }_{1} \rho}, \quad g_{2}=\frac{{ }_{1} \rho_{12}}{{ }_{1} \rho}, g_{3}=\frac{{ }_{1} \rho_{22}}{{ }_{1} \rho}, \\
& x_{1}=\left(\frac{{ }_{1} V_{0}}{{ }_{1} V_{1}}\right)^{2}, \quad y_{1}=\left(\frac{{ }_{1} V_{0}}{{ }_{1} V_{2}}\right)^{2}, z_{1}=\left(\frac{{ }_{1} V_{0}}{{ }_{1} V_{3}}\right)^{2}, x_{2}=\left(\frac{{ }_{1} V_{0}}{{ }_{2} V_{1}}\right)^{2}, \quad y_{2}=\left(\frac{{ }_{1} V_{0}}{{ }_{2} V_{2}}\right)^{2}, \quad z_{2}=\left(\frac{{ }_{1} V_{0}}{{ }_{2} V_{3}}\right)^{2}, \quad m=\frac{C}{{ }_{1} C_{0}},
\end{aligned}
$$

where $m$ is non-dimensional phase velocity and

$$
{ }_{1} H={ }_{1} P+2{ }_{1} Q+{ }_{1} R,{ }_{1} \rho={ }_{1} \rho_{11}+2{ }_{1} \rho_{12}+{ }_{1} \rho_{22}, \quad{ }_{1} C_{0}^{2}=\frac{{ }_{1} N}{{ }_{1} \rho}, \quad{ }_{1} V_{0}^{2}=\frac{{ }_{1} H}{{ }_{1} \rho} \text {. }
$$

Non-dimensional phase velocity is calculated for two types of composite cylinders, namely composite cylinder-I and composite cylinder-II each for a pervious and an impervious surface. Composite cylinder-I consists of core made up of sandstone saturated 
with water (Yew and Jogi, 1976) and casing is made up of sandstone saturated with kerosene (Fatt, 1957), where as in composite cylinder-II, the core is sandstone saturated with kerosene and casing is sandstone saturated with water. The physical parameters of these poroelastic composite materials following equation (44) are given in Table 1.

Table - 1 Physical parameters of poroelastic composite materials

\begin{tabular}{|c|c|c|c|c|c|c|c|c|c|c|}
\hline $\begin{array}{c}\text { Material } \\
\text { Parameters }\end{array}$ & $\mathrm{a}_{1}$ & $\mathrm{a}_{2}$ & $\mathrm{a}_{3}$ & $\mathrm{a}_{4}$ & $\mathrm{~d}_{1}$ & $\mathrm{~d}_{2}$ & $\mathrm{~d}_{3}$ & $\mathrm{x}_{2}$ & $\mathrm{y}_{2}$ & $\mathrm{z}_{2}$ \\
\hline $\begin{array}{c}\text { Composite } \\
\text { Cylinder-I }\end{array}$ & 0.445 & 0.034 & 0.015 & 0.123 & 0.887 & -0.001 & 0.099 & 1.863 & 8.884 & 7.183 \\
\hline $\begin{array}{c}\text { Composite } \\
\text { Cylinder-II }\end{array}$ & 1.819 & 0.011 & 0.054 & 0.780 & 0.891 & 0 & 0.125 & 0.489 & 2.330 & 1.142 \\
\hline
\end{tabular}

\begin{tabular}{|c|c|c|c|c|c|c|c|c|c|}
\hline $\mathrm{b}_{1}$ & $\mathrm{~b}_{2}$ & $\mathrm{~b}_{3}$ & $\mathrm{~b}_{4}$ & $\mathrm{~g}_{1}$ & $\mathrm{~g}_{2}$ & $\mathrm{~g}_{3}$ & $\mathrm{x}_{1}$ & $\mathrm{y}_{1}$ & $\mathrm{z}_{1}$ \\
\hline 0.960 & 0.006 & 0.028 & 0.412 & 0.877 & 0 & 0.123 & 0.913 & 4.347 & 2.129 \\
\hline 0.843 & 0.065 & 0.028 & 0.234 & 0.901 & -0.001 & 0.101 & 0.999 & 4.763 & 3.851 \\
\hline
\end{tabular}

\section{Results and Discussion}

For given poroelastic parameters, the frequency equations when non-dimensionalized using equation (44), constitute a relation between non-dimensional phase velocity and non-dimensionalized wavenumber. Different values of $\mathrm{a} / \mathrm{r}_{1}$ and $\mathrm{r}_{2} / \mathrm{a}$, viz., 1.1 and 3 are taken for numerical computation. For a poroelastic shell made of single material, the value (ratio of outer radius to inner radius) 1.1 represents thin poroelastic shell, whereas the value 3 represents thick poroelastic shell.

Figures 1-4 depict phase velocity of vibrations of poroelastic composite hollow cylinders I and II for different combinations of thin and thick shells for a pervious and an impervious surfaces. In Fig.1, phase velocity for thin core and thin casing has been plotted. The phase velocity of pervious and impervious surfaces for each of the cylinders I and II is almost same. The phase velocity of cylinder II is more than that of cylinder I when wave number is between 0 and 4 . It is clear that the phase velocity of cylinder I is steady. Fig. 2 shows the phase velocity for thin core and thick casings. The phase velocity is same for both pervious and impervious surfaces in case of composite cylinder I, whereas for cylinder II it is true when wave number is greater than 3 . The variation of phase velocity for thin casing and thick core is shown in Fig. 3. The phase velocity is same for pervious and impervious surfaces when the wave number is less than 2 and greater than 7 in case of cylinder I, whereas in case of cylinder II it is true when the wave number is greater than 2. Fig. 4 shows the phase velocity for thick core and thick casings. The phase velocity is same for pervious and impervious surfaces in case of cylinder I, whereas in case of cylinder II the phase velocity of impervious surface is slightly more than that of pervious surface.

Figures. 5-6 depict phase velocity for poroelastic casing when the solid is rigid. In particular, thin casing is considered in Fig.5, whereas thick casing is considered in Fig. 6. In case of thin casing, the phase velocity is more for cylinder I when the wave number is between 0 and 4 . The phase velocity is constant for an impervious surface for both the cylinders when the wave number is greater than 4 . There is a sudden increase in phase velocity when wave number is 8 for cylinder I in case of thick casing. The variation in phase velocity for poroelastic core when it is clamped along its outer surface is shown in Figs. 7-8. In particular, thin core is considered in Fig.7, whereas thick core is considered in Fig.8. From Fig. 7, it is clear that the phase velocity is same for both cylinders for each pervious and impervious surface. The phase velocity is same for both cylinders when wave number is between 0 and 2. Also, the phase velocity is maximum when wave number is 1 . In case of thick core, phase velocity is same for both cylinders when wave number is between 0 and 1 .

The variation in phase velocity for poroelastic composite bore is shown in Figs. 9-10. In particular, composite bore with thin core is considered in Fig. 9, whereas composite bore with thick core is considered in Fig.10. From Fig. 9, it is clear that the phase velocity for composite bore I higher than that of cylinder II when wave number is less than 4.5 for an impervious surface. Also, the maximum phase velocity is observed when wave number is 8 for an impervious surface for composite bore I.

\section{Conclusion}

A study on vibrations in a poroelastic composite hollow cylinder and composite bore has been done, also the effects of rigidity and infinite wavelength have been observed, and these lead to the following conclusions:

(i) Plane-strain vibrations and longitudinal shear vibrations of poroelastic composite hollow cylinder are uncoupled when wavelength is infinite each for a pervious and an impervious surface. Similar observation has been found in the case of composite bore.

(ii) The frequency equation of longitudinal shear vibrations of poroelastic composite hollow cylinder is independent of nature of surface for infinite wavelength. 
(iii) The vibrations of poroelastic composite hollow cylinder related to core and casing for pervious surface are uncoupled when the solid in casing is rigid. In particular, in case of core the vibrations are observed when it clamped along its outer surface.

(iv) The phase velocity of poroelastic composite hollow cylinder is almost same for pervious and impervious surfaces for both cylinders I and II in cases of thin core and thin casing as well as thin core and thick casing. Thus, the phase velocity is independent of nature of surface when the core is thin.

(v) Variations in phase velocity is more in thick core than thin core when core is clamped along its outer surface.

The thermal effect on composite cylinder can be discussed; also similar study can be done on composite cylinders and finite composite poroelastic cylinders in various dimensions and on composite spheres.

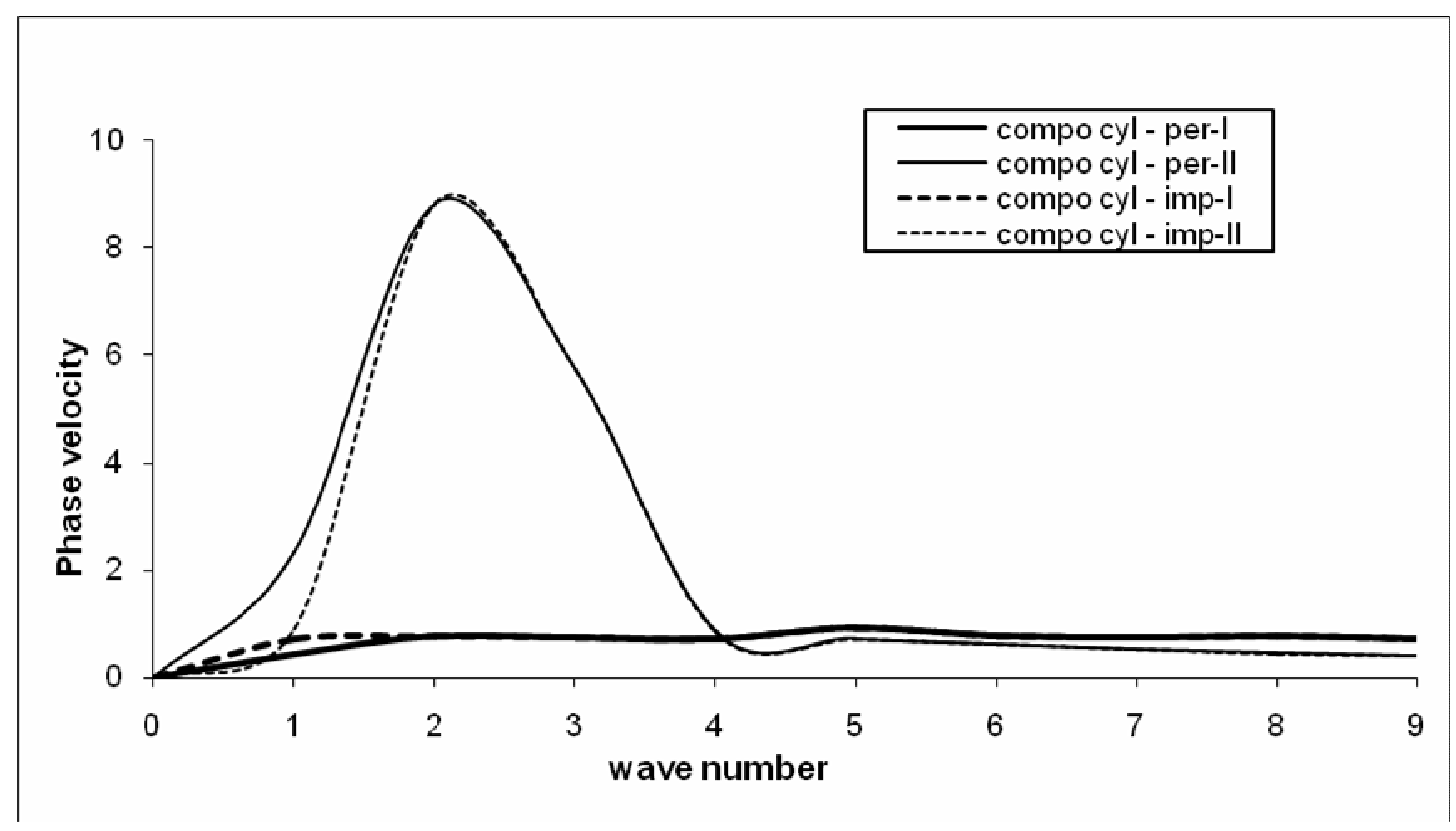

Fig.1 Variation of phase velocity with the wave number - poroelastic composite hollow cylinder Thin core and thin casing

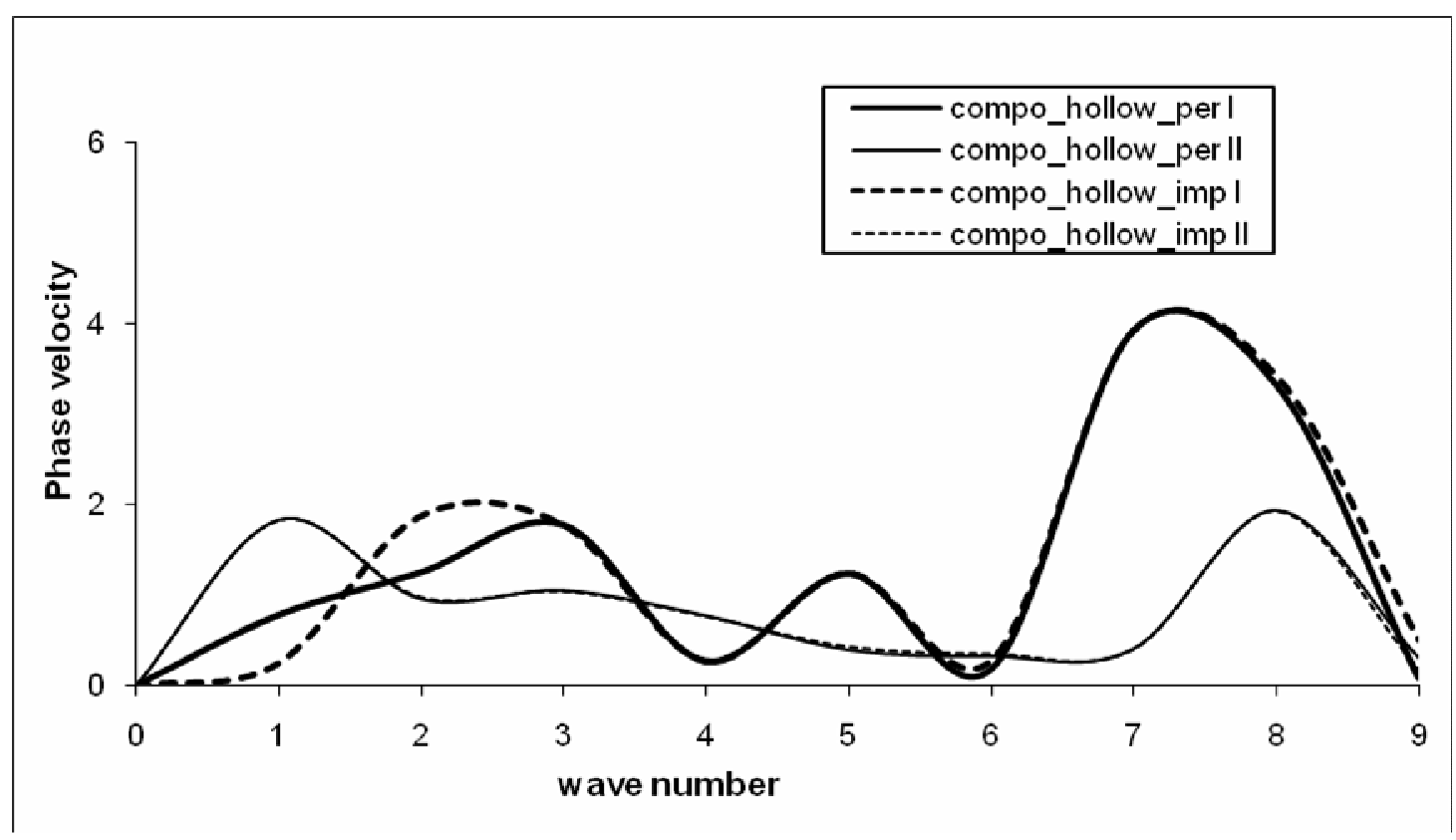

Fig.2 Variation of phase velocity with the wave number - poroelastic composite hollow cylinder -

Thin core and thick casing 


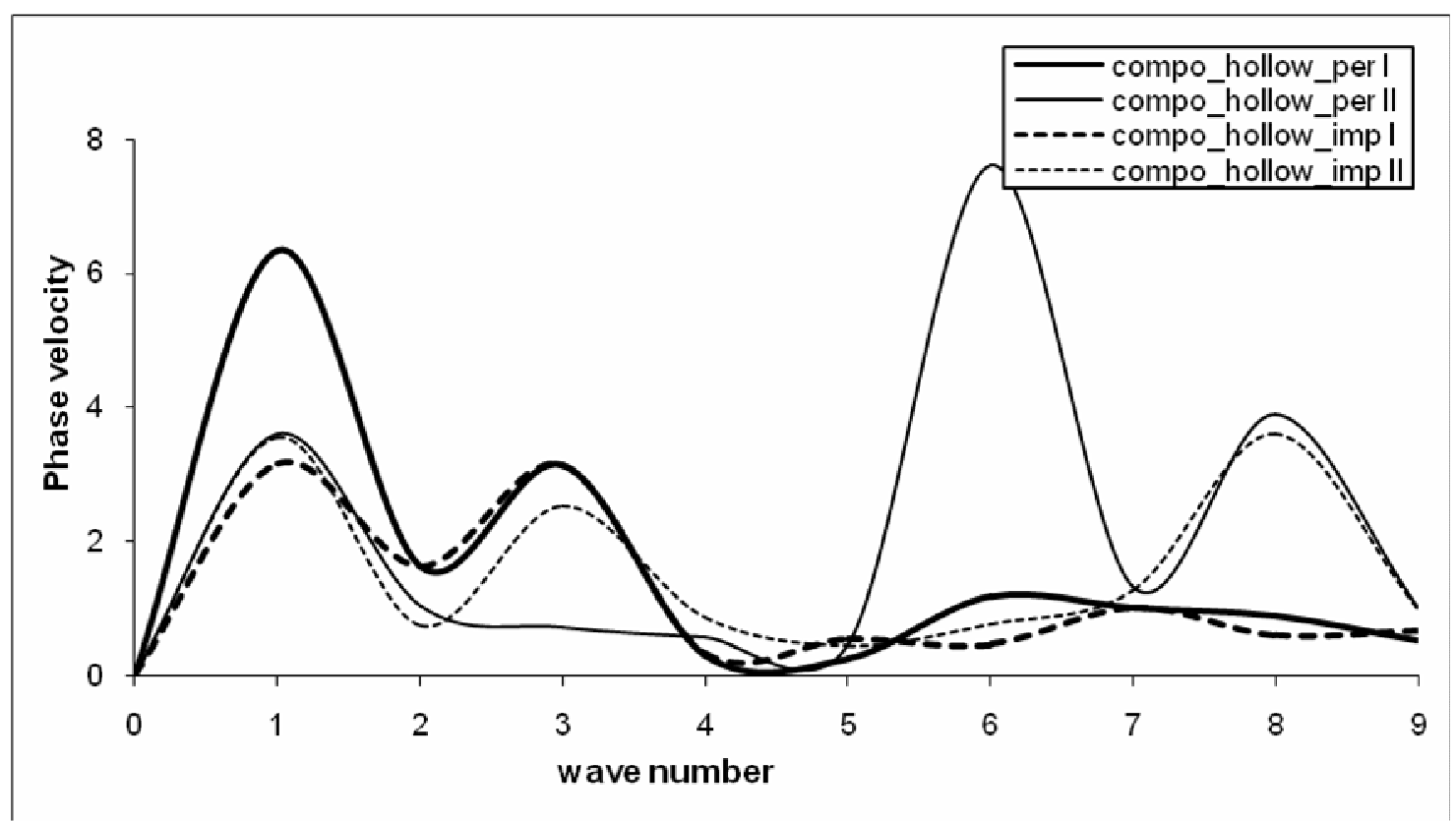

Fig.3 Variation of phase velocity with the wave number - poroelastic composite hollow cylinder Thick core and thin casing

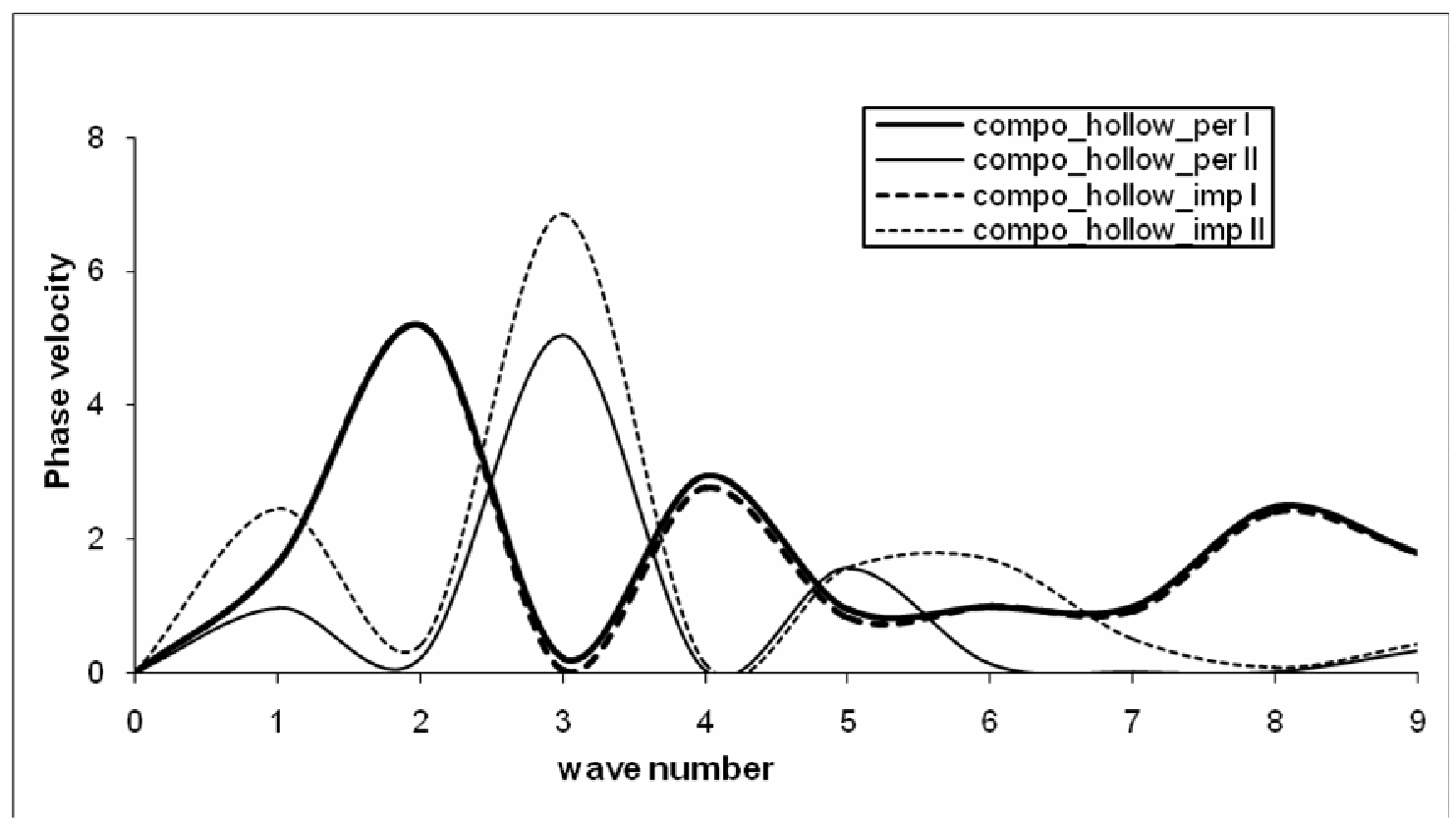

Fig.4 Variation of phase velocity with the wave number - poroelastic composite hollow cylinder -

Thick core and thick casing 


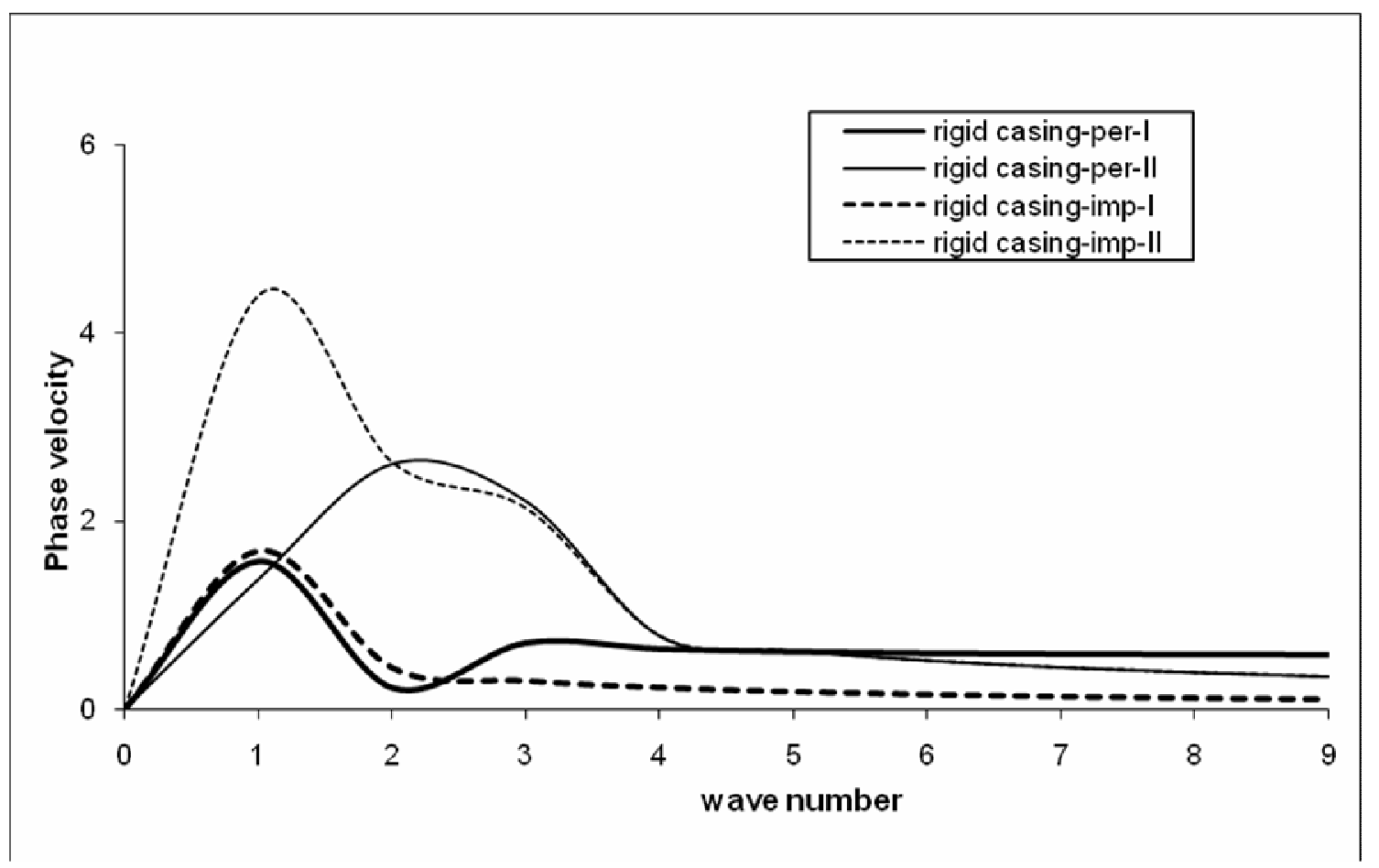

Fig. 5 Variation of phase velocity with the wave number - poroelastic rigid casing - Thin shell

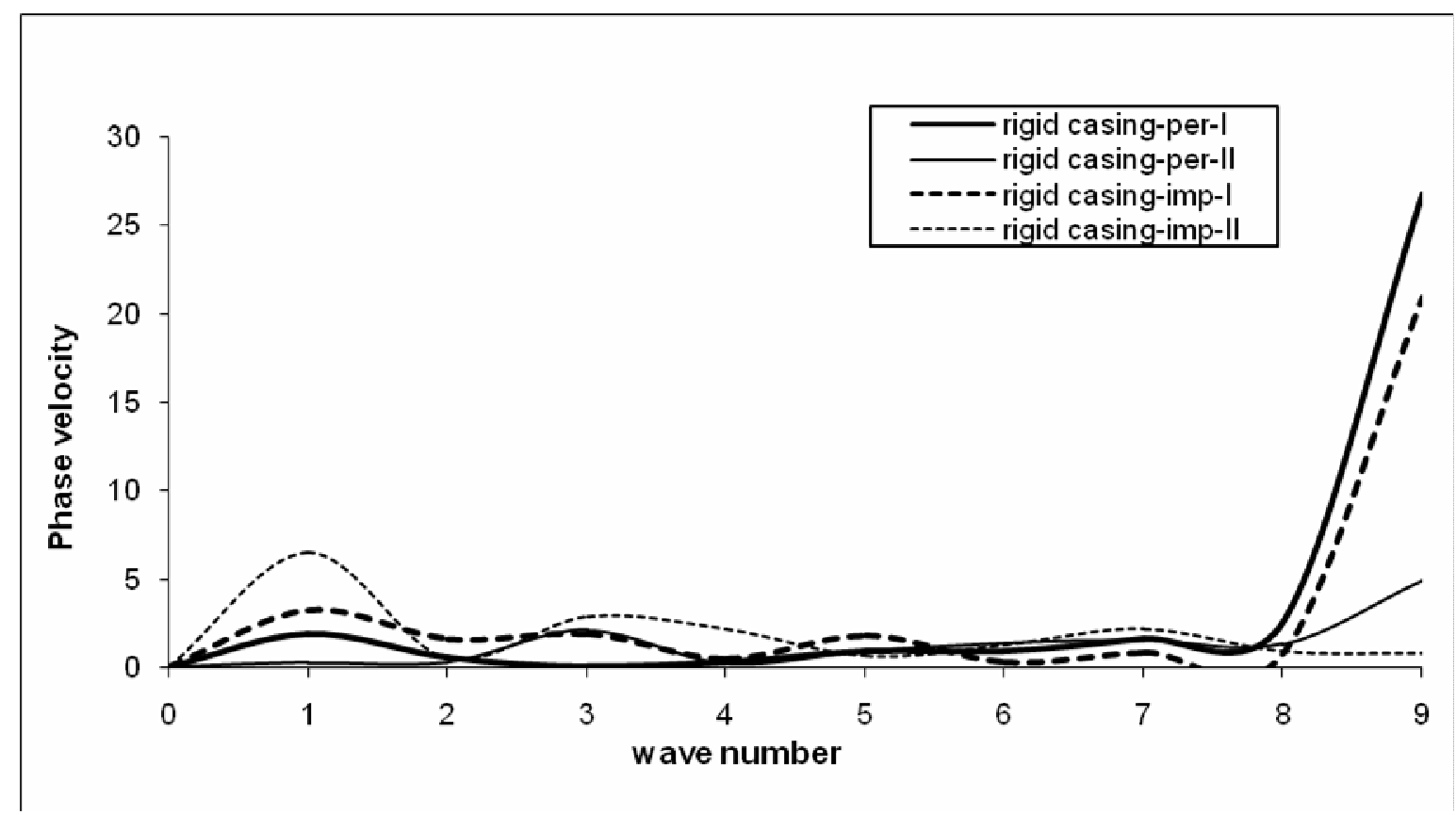

Fig. 6 Variation of phase velocity with the wave number - poroelastic rigid casing - Thick shell 


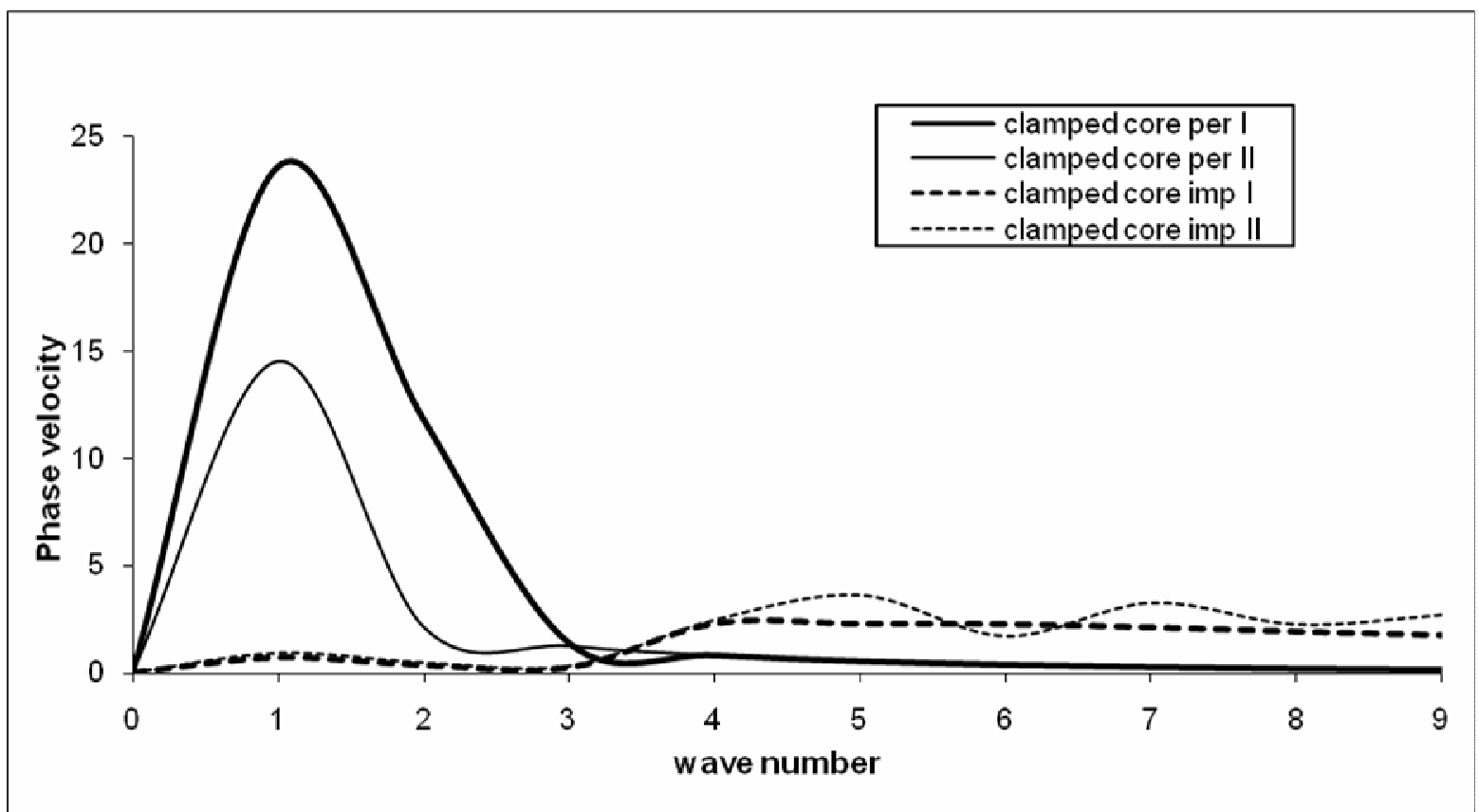

Fig.7 Variation of phase velocity with the wave number - poroelastic shell clamped along its outer surface - Thin shell

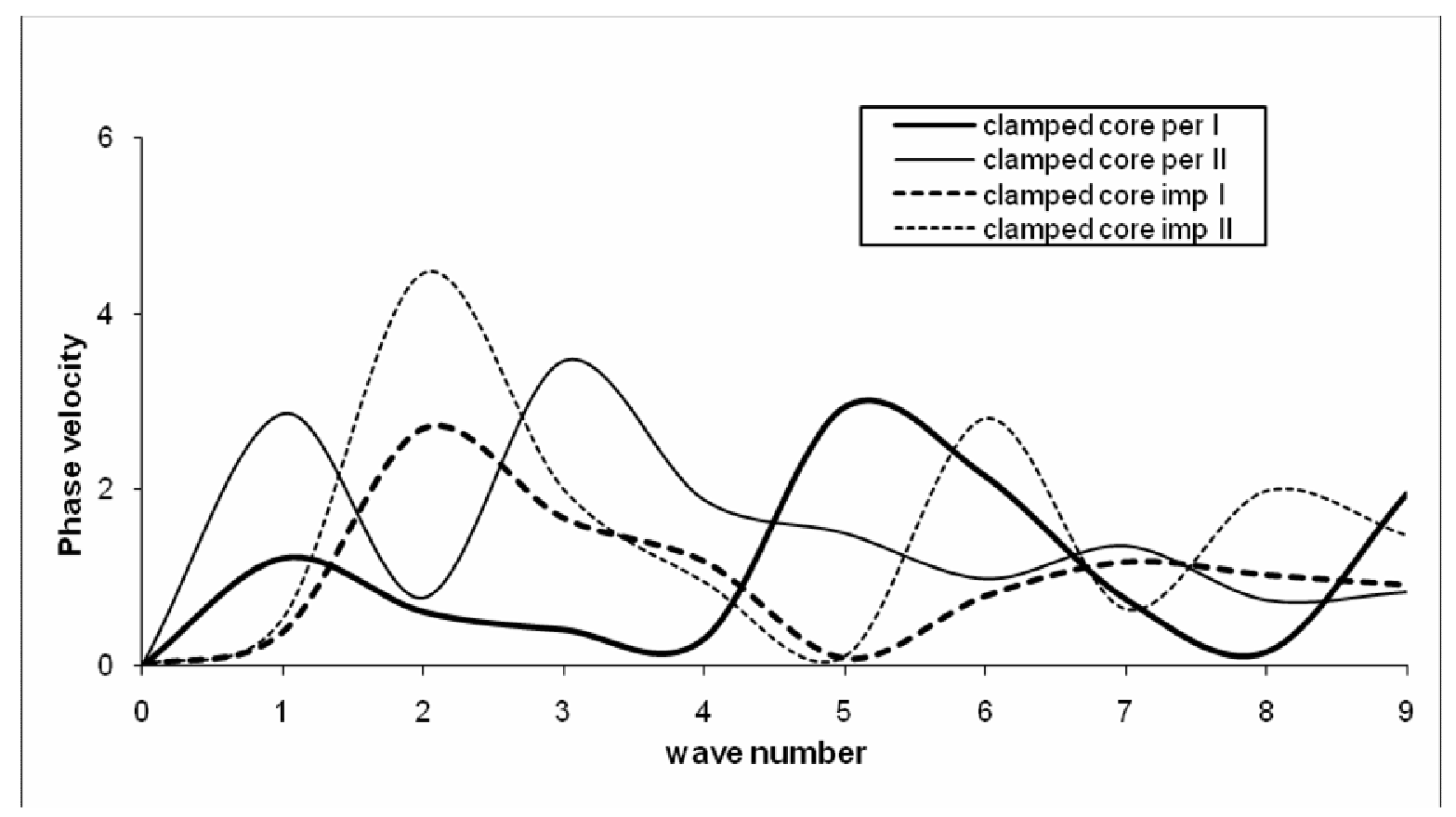

Fig. 8 Variation of phase velocity with the wave number - poroelastic shell clamped along its outer surface - Thick shell 


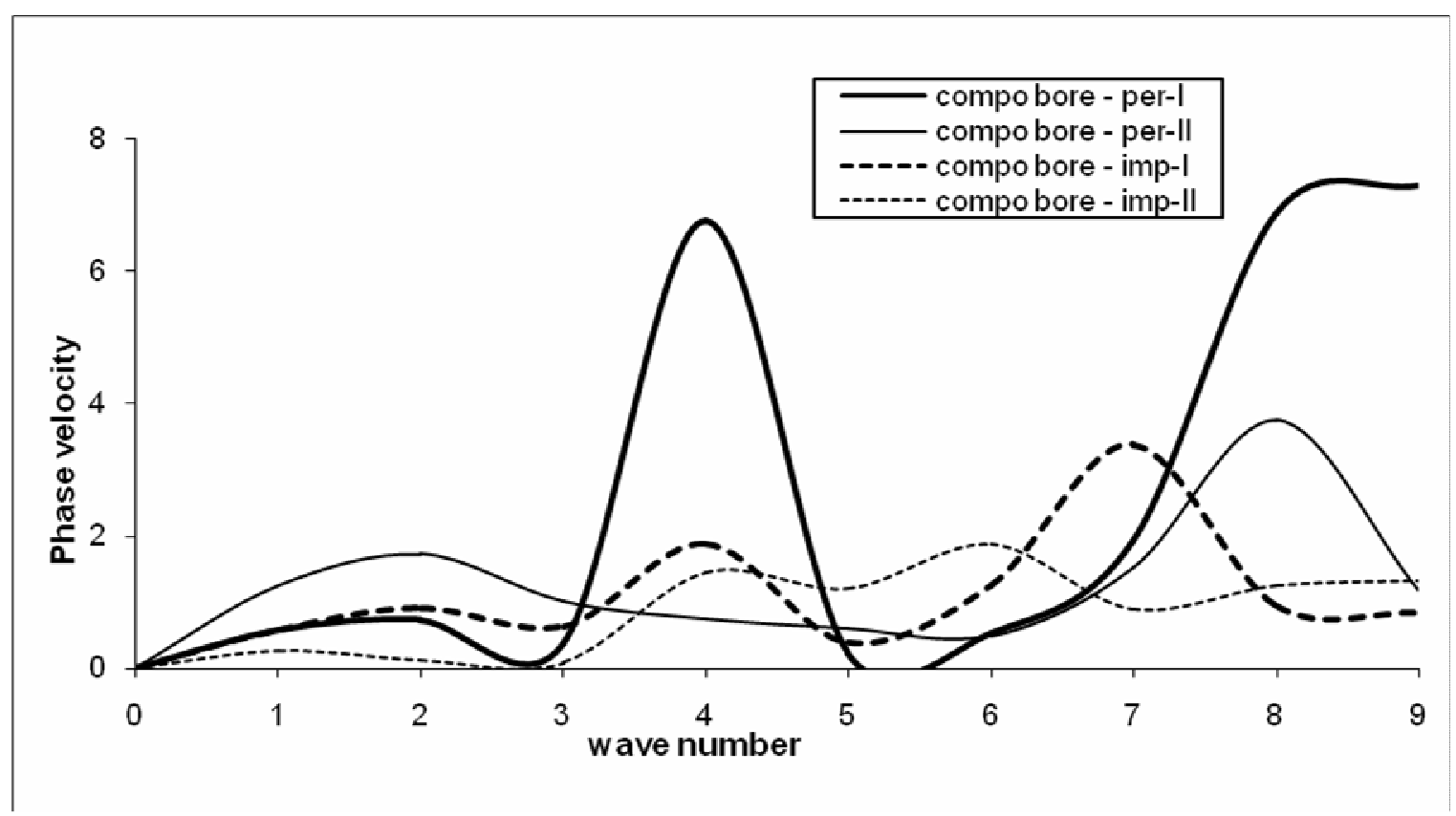

Fig.9 Variation of phase velocity with the wave number - poroelastic composite bore Thin core

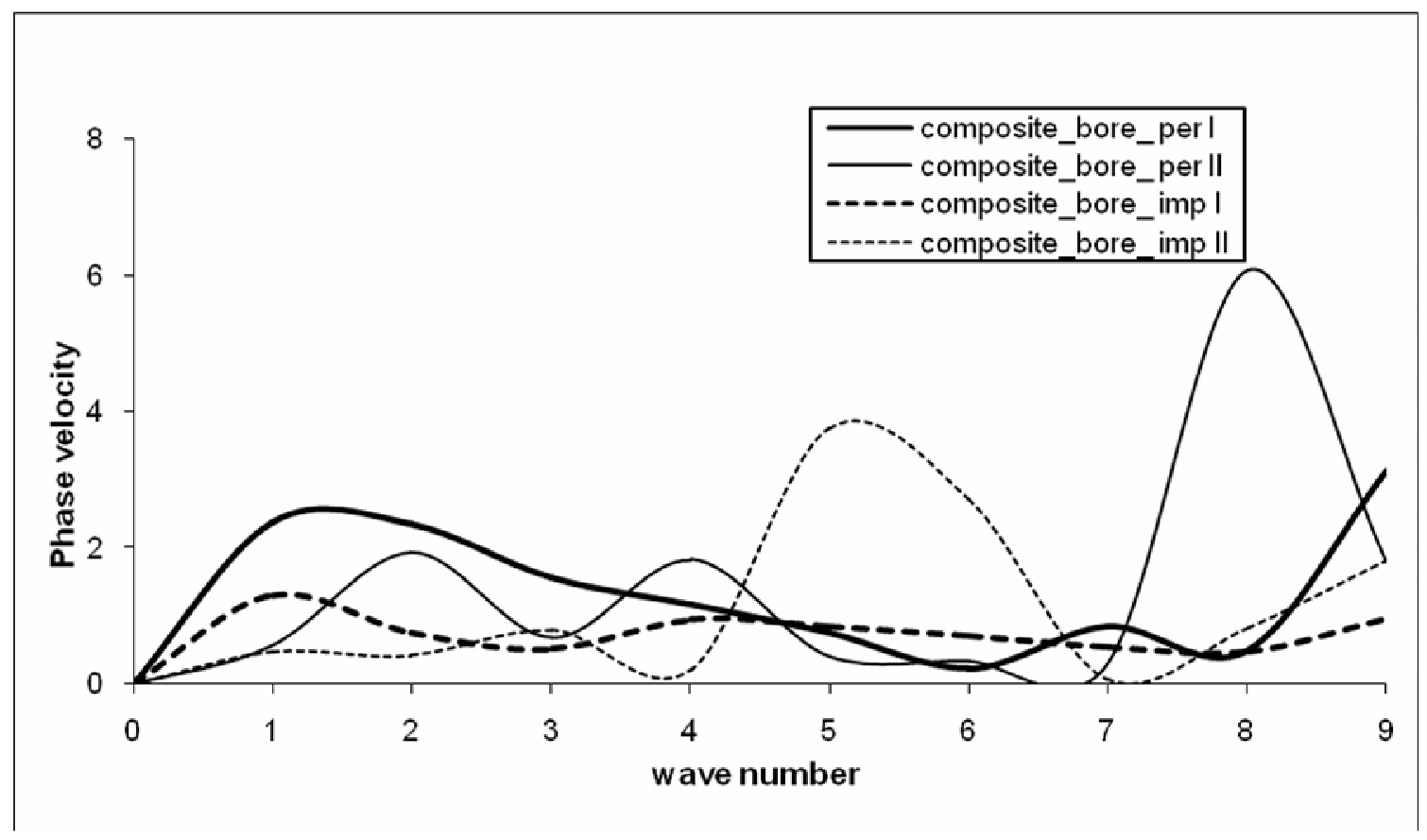

Fig.10 Variation of phase velocity with the wave number - poroelastic composite bore Thick core

\section{Nomenclature}

$\left.\begin{array}{l}{ }_{1} A,{ }_{1} N,{ }_{1} Q,{ }_{1} R \\ { }_{2} A,{ }_{2} N,{ }_{2} Q,{ }_{2} R\end{array}\right\}$ - Poroelastic constants a - iterface radius 


$$
\begin{aligned}
& \mathrm{b} \text { - dissipation coefficient } \\
& \text { e - dilatation of solid } \\
& J_{\mathrm{n}}-\text { Bessel function of first kind of order } n \\
& \mathrm{r}_{1}-\text { inner radius of core } \\
& \mathrm{r}_{2} \text { - outer radius of casing } \\
& \mathrm{s}-\text { liquid pressure } \\
& \mathrm{t} \text { - time } \\
& \mathbf{U} \text { - liquid displacement } \\
& \mathbf{u} \text { - solid displacement } \\
& { }_{1} V_{1,2} V_{1} \text { - dilatational wave velocities of first kind } \\
& { }_{1} V_{2,2} V_{2} \text { - dilatational wave velocities of second kind } \\
& Y_{\mathrm{n}} \text { - Bessel function of second kind of order }
\end{aligned}
$$

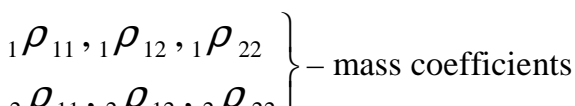

$$
\begin{aligned}
& \left.{ }_{2} \rho_{11},{ }_{2} \rho_{12},{ }_{2} \rho_{22}\right\} \\
& { }_{1} \sigma_{i j, 2} \sigma_{i j}-\text { stresses } \\
& \varepsilon \text {-dilatation of liquid } \\
& k \text { - wavenumber } \\
& m \text { - non-dimensional phasevelocity } \\
& \nabla^{2}-\text { Laplacian operator }
\end{aligned}
$$

\section{References}

Abousleiman, Y.N., and Cui. L., 1998. Poroelastic solutions in transversely isotropic media for wellbore cylinders, International Journal of Solids and Structures, Vol.35, pp. 4905-4929.

Ahmed Shah, S., 2011. Flexural wave propagation in coated poroelastic cylinders with reference to fretting fatigue, Journal of Vibration and Control, Vol. 17, pp.1049-1064.

Ahmed Shah, S., and Tajuddin, M., 2009. Axial symmetric vibrations of finite composite poroelastic cylinders, International Journal of Applied Mechanics and Engineering, Vol.14, pp.865-877.

Ahmed Shah, S., and Tajuddin, M., 2011. Torsional vibrations of thick-walled hollow poroelastic spheres, Bull. Cal. Math. Soc., Vol.103, pp.161-170.

Biot, M.A., 1956. The theory of propagation of elastic waves in fluid-saturated porous solid, Journal of the Acoustical Society of America, Vol. 28, pp.168-178.

Baltrukonis, J. H., Gottenberg, W.G., and Schreiner, R.N., 1961. Axial-shear vibrations of an infinitely long composite circular cylinder, Journal of the Acoustical Society of America, Vol. 33, pp.1447-1457.

Chen, W. Q., and Ding, H. J., 2001. Free vibration of multi-layered spherically isotropic hollow spheres, International Journal of Mechanical Sciences, Vol. 43, pp. 667-680.

Cui, L., Cheng. A. H. D., and Abousleiman. Y., 1997. Poroelastic solutions of an inclined borehole, J. Appl. Mech., Vol. 64, pp. 32-38.

Fatt, I., 1959. The Biot-willis elastic coefficients for a sandstone, Journal of Applied Mechanics, Vol. 26, pp. 296-297.

Malla Reddy, P., and Tajuddin, M., 2010. Axially symmetric vibrations of composite poroelastic cylinders in the context of fretting fatigue, Special Topics and Reviews in Porous Media, Vol. 1, pp. 311-320.

McFadden, J. A., 1954. Radial vibrations of thick-walled hollow cylinders, Journal of the Acoustical Society of America, vol. 26, pp. 714-715.

Paul, S., 1974. A note on the Radial vibrations of a sphere of poroelastic material. Indian Journal of Pure and Applied Mathematics, Vol. 7, pp. 469-475.

Tajuddin, M., and Ahmed shah, S., 2010. Radial vibrations of thick-walled hollow poroelastic cylinders, Journal of Porous Media, Vol. 13, pp. 307-318.

Tajuddin, M., Nageswara Nath, C., and Manoj Kumar, J., 2011. Axial-shear vibrations of an infinitely long poroelastic composite circular cylinder, Special Topics \& Reviews in Porous Media, Vol. 2, pp. 133-143.

Stavsky,Y., and Greenberg, J. B., 2003. Radial vibrations of orthotropic laminated hollow spheres, Journal of the Acoustical Society of America, Vol. 113, pp. 847-857

Yew, C.H., and Jogi, P.N., 1976. Study of wave motions in fluid-saturated porous rocks, Journal of the Acoustical Society of America, Vol. 60, pp. 2-8. 


\section{Biographical notes}

J. Manoj Kumar is in the ${ }^{1}$ Department of Mathematics, BVRIT College of Engineering for Women, Hyderabad - 500 090, India

Dr.S.Ahmed Shah is a Professor in the Department of Mathematics, Deccan College of Engineering and Technology Hyderabad, India. He has more than 18 years of teaching experience. He published papers in referred national and international journals and has more than 10 years of research experience. His area of research includes stress wave propagation in poroelastic solids.

C. Nageswara Nath is in the ${ }^{3}$ Department of Mathematics, CMR Technical Campus, Hyderabad - 501 401, India

Received May 2015

Accepted December 2015

Final acceptance in revised form December 2015 